\title{
Design, synthesis, antitubercular and antiviral properties of new spirocyclic indole derivatives
}

\author{
Gökçe Cihan-Üstündağ ${ }^{1}$ (D) - Lieve Naesens ${ }^{2} \cdot$ Dilek Şatana $^{3} \cdot$ Gonca Erköse-Genç ${ }^{3}$ Emel Mataracı-Kara ${ }^{4}$. \\ Gültaze Çapan ${ }^{1}$
}

Received: 12 March 2019 / Accepted: 12 June 2019 / Published online: 17 July 2019

(c) Springer-Verlag GmbH Austria, part of Springer Nature 2019

\begin{abstract}
A series of indole-based spirothiazolidinones have been designed, synthesized and evaluated, in vitro, for their antitubercular, antiviral, antibacterial, and antifungal activities. The structures of the new compounds were established by IR, ${ }^{1} \mathrm{H}$ NMR, ${ }^{13} \mathrm{C}$ NMR (proton decoupled, APT, and DEPT), electrospray ionization mass spectrometry, and microanalysis. Compounds bearing a phenyl substituent at position 8 of the spiro ring, exhibited significant antitubercular activity against Mycobacterium tuberculosis $\mathrm{H} 37 \mathrm{Rv}$ ATCC 27294 at concentrations of 3.9 and $7.8 \mu \mathrm{M}$. Still, some of the tested compounds displayed activity on mycobacteria with MIC values of 16 and $31 \mu \mathrm{M}$. Four of the indole-spirothiazolidinone derivatives were found to be moderately active against Punta Toro virus, yellow fever virus or Sindbis virus in Vero cells. The antiviral $\mathrm{EC}_{50}$ values were in the range of 1.9-12 $\mu \mathrm{M}$ and the selectivity index (ratio of cytotoxic to antivirally effective concentration) was above 10 in some cases. The most potent effect was seen with the compound that is methylated at positions 2 and 8 of the spirothiazolidinone system.
\end{abstract}

\section{Graphic abstract}
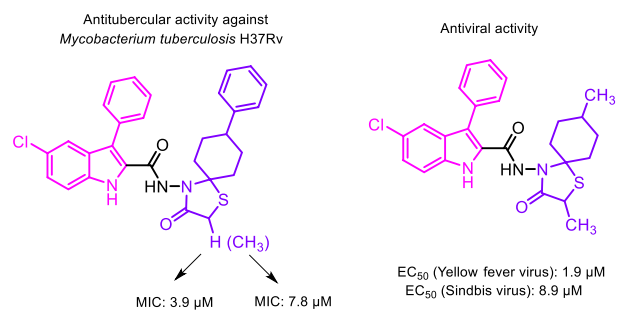

Keywords Heterocycles $\cdot$ Spirothiazolidinone $\cdot$ Antitubercular activity $\cdot$ Antiviral activity $\cdot$ Cytotoxicity $\cdot$ Drug research

\section{Introduction}

Electronic supplementary material The online version of this article (https://doi.org/10.1007/s00706-019-02457-9) contains supplementary material, which is available to authorized users.

Gökçe Cihan-Üstündağ

gokcechn@istanbul.edu.tr

1 Department of Pharmaceutical Chemistry, Faculty of Pharmacy, Istanbul University, Istanbul 34116, Turkey

2 Department of Microbiology, Immunology and Transplantation, Rega Institute for Medical Research, KU Leuven, 3000 Louvain, Belgium
Tuberculosis (TB) is a highly infectious disease caused by the bacillus Mycobacterium tuberculosis. For the past 5 years, it has been the leading cause of mortality from a

3 Department of Microbiology and Clinical Microbiology, Istanbul Faculty of Medicine, Istanbul University, Istanbul 34116, Turkey

4 Department of Pharmaceutical Microbiology, Faculty of Pharmacy, Istanbul University, 34116 Istanbul, Turkey 
single infectious disease, ranking above HIV/AIDS [1]. Major problems associated with the currently available TB treatment include long treatment duration, inadequate compliance, concurrent HIV infection, and increasing incidence of multidrug-resistant (MDR) and extensively drug-resistant (XDR) tuberculosis [2-5]. This emergence of difficult to treat strains necessitates the discovery and development of novel antitubercular drugs. After this domain has been inactive for several decades, two new drugs became available, i.e., the mitochondrial ATP synthase inhibitor bedaquiline and mycolic acid biosynthesis inhibitor delamanid, which received accelerated approval for the treatment of MDR tuberculosis in 2012 and 2014, respectively [6]. Besides, diverse novel drug candidates are in preclinical or clinical development [6-8].

Indole-2-carboxamides incorporating an alicyclic system (Fig. 1a) have been extensively studied by different research groups [9-14]. This type of compounds was found to be highly active against both drug-susceptible and drugresistant strains of Mycobacterium tuberculosis by acting on the MmpL3 transporter protein. In previous investigations, we have identified the indole-spirothiazolidinone system (Fig. 1b) as a promising scaffold against the Mycobacterium tuberculosis H37Rv strain $[15,16]$. Some of these analogues exhibited in vitro antitubercular activity with GI (growth inhibition) values of $91-95 \%$ at a MIC (minimum inhibitory concentration) value of $6.25 \mu \mathrm{g} / \mathrm{cm}^{3}$. Recently, we reported on the synthesis of novel 5-fluoro-3-phenyl- $1 H$-indole derivatives containing a 4-thiazolidinone nucleus (instead of spirothiazolidinone system) [17]. Two molecules (Fig. 1c) displayed notable antitubercular activity at concentrations tenfold lower than those that produced cytotoxicity in mammalian cell lines.

Furthermore, several spirothiazolidinone compounds synthesized in our laboratory were found to be efficient inhibitors of membrane fusion mediated by influenza virus hemagglutinin (HA) [18-20]. As demonstrated in Fig. 1d, these compounds have a similar backbone structure, consisting of an aromatic/polycyclic ring linked to a non-aromatic spiro system via an amide bridge. Some analogues displayed low micromolar activity against the influenza A/H3N2 subtype with a favorable selectivity index.

Based on these insights and our objective to optimize the antimicrobial activity of indolyl thiazolidinones and spirothiazolidinones, we here report the chemical synthesis, structural characterization and in vitro antitubercular, antiviral, antibacterial, and antifungal evaluation of new 5-chloro-3-phenyl- $N$-(2,7,8,9-substituted/nonsubstituted3-oxo-1-thia-4-azaspiro[4.4]nonan/[4.5]decan-4-yl)- $1 H$-indole-2-carboxamides $\mathbf{4 a - 4 i , 5 a - 5 h ~ ( F i g . ~ 1 e ) . ~}$

\section{Results and discussion}

\section{Chemistry and structural characterization}

The synthetic pathways for the preparation of the spirothiazolidinones 4a-4i and 5a-5h are illustrated in Scheme 1 . Thus, the diazonium salt, formed by the reaction of 4-chloroaniline with $\mathrm{NaNO}_{2}$ and $\mathrm{HCl}$, was reacted with ethyl 2-benzyl-3-oxobutanoate to obtain compound 1 [21] according
$(\mathrm{F}, \mathrm{C})$

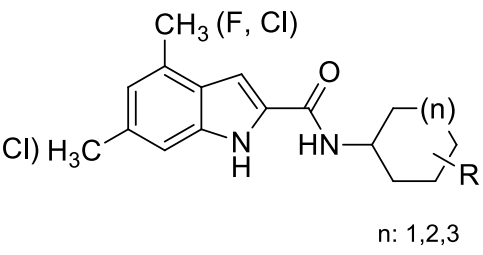

(a)<smiles>[R]C1CCC2(CC1)SC(C)C(=O)N2NC(=O)c1[nH]c2ccc(C)cc2c1C</smiles>

(b)<smiles>CCCCCCCc1c(C(=O)NN2C(=O)CSC2c2ccc(C(=O)[N+](=O)[O-])cc2)[nH]c2ccc(F)cc12</smiles>

(c)

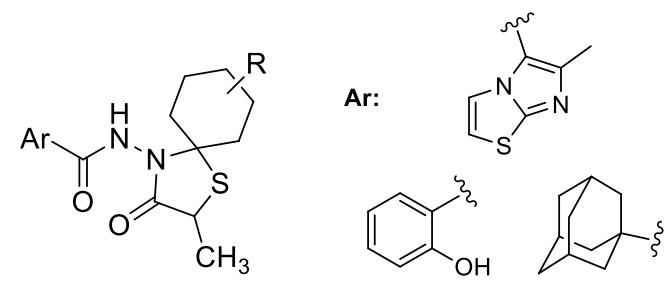

(d)<smiles>[R]CC1(CC)SC(C)C(=O)N1NC(=O)c1[nH]c2ccc(Cl)cc2c1C</smiles>

(e) $\mathrm{n}: 1,2$

Fig. 1 Structures of $\mathbf{a}, \mathbf{b}, \mathbf{c}$ indole-2-carboxamide-based antitubercular agents and $\mathbf{d}$ the spirothiazolidinone compounds acting as influenza A virus fusion inhibitors 


\section{Scheme 1}<smiles>CC(C)C</smiles><smiles>CCOC(=O)/C(CCc1ccccc1)=N\Nc1ccc(Cl)cc1</smiles>

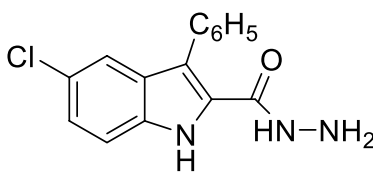

3

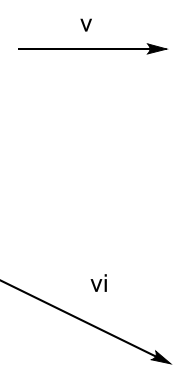

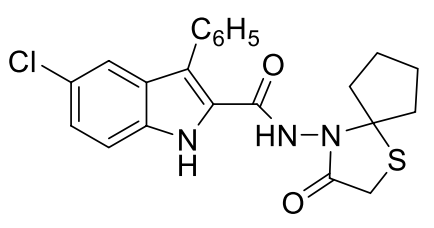

$4 a$

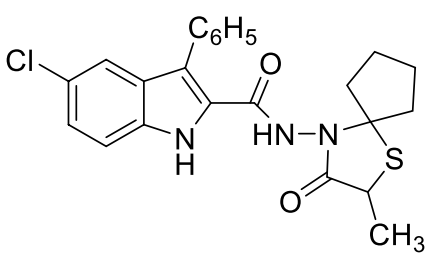<smiles>[C]1CCCC1</smiles><smiles>CCOC(=O)c1[nH]c2ccc(Cl)cc2c1C(C)(C)CC</smiles><smiles>[R]C1CC2(CC([R])C([R])C([R])([R])C2)SCC1=O</smiles>

$4 b-4 i$<smiles>[R]C1CC2(CC([R])C([R])([R])C2)SC(C)C1=O</smiles>

5b-5h

(i) $7 \% \mathrm{NaNO}_{2}, \mathrm{EtOH}$, conc. $\mathrm{HCl}, 0{ }^{\circ} \mathrm{C}$; (ii) ethyl 2-benzyl-3-oxo-butanoate, $\mathrm{KOH}, \mathrm{EtOH}, 0{ }^{\circ} \mathrm{C}$; (iii) conc. $\mathrm{HCl}$, reflux, 4 h; (iv) $\mathrm{H}_{2} \mathrm{NNH}_{2} \cdot \mathrm{H}_{2} \mathrm{O}$, EtOH, reflux, $6 \mathrm{~h}$; (v) cyclopentanone/3,4,5-(non)substituted cyclohexanone, mercaptoacetic acid, dry toluene, reflux, 5-6 h; (vi) cyclopentanone/3,4,5-(non)substituted cyclohexanone, 2-mercaptopropionic acid, dry toluene, reflux, 5-6 h. 4b, 5b: $R, R^{1}, R^{2}, R^{3}=H ; 4 c, 5 c: v R=C H_{3} ; R^{1}, R^{2}, R^{3},=H ; 4 d, 5 d: R^{1}=C H_{3} ; R, R^{2}, R^{3}=H$;

4e, 5e: $\mathrm{R}^{1}=\mathrm{C}_{2} \mathrm{H}_{5} ; \mathrm{R}, \mathrm{R}^{2}, \mathrm{R}^{3}=\mathrm{H} ; \mathbf{4 f}, \mathbf{5 f}: \mathrm{R}^{1}=\mathrm{C}_{3} \mathrm{H}_{7} ; \mathrm{R}, \mathrm{R}^{2}, \mathrm{R}^{3}=\mathrm{H} ; \mathbf{4 g}, \mathbf{5 g}: \mathrm{R} \mathrm{R}^{2}, \mathrm{R}^{3}=\mathrm{CH}_{3} ; \mathrm{R}^{1}=\mathrm{H} ; \mathbf{4 h}, \mathbf{5 h}: \mathrm{R}^{1}=\mathrm{C}_{6} \mathrm{H}_{5} ; \mathrm{R}, \mathrm{R}^{2}, \mathrm{R}^{3}=\mathrm{H}$; $4 i: \mathrm{R}^{1}=\mathrm{C}\left(\mathrm{CH}_{3}\right)_{3} ; \mathrm{R}, \mathrm{R}^{2}, \mathrm{R}^{3}=\mathrm{H}$

to the Japp-Klingemann reaction. The Fischer indole synthesis was carried out in acidic medium to cyclize $\mathbf{1}$ into ethyl 5-chloro-3-phenyl- $H$-indole-2-carboxylate 2 [22]. Subsequent exposure of $\mathbf{2}$ to an excess of hydrazine hydrate afforded compound 3 [23]. The target spirocyclic compounds $4 \mathbf{a}-\mathbf{4 i}, \mathbf{5 a}-\mathbf{5 h}$ were synthesized by treatment of the key intermediate $\mathbf{3}$ with appropriate cyclic ketones and mercapto acids in a one-pot reaction [15].

The structures of the new compounds were characterized by IR, ${ }^{1} \mathrm{H}$ NMR, ${ }^{13} \mathrm{C}$ NMR (proton decoupled, APT, and DEPT), electrospray ionization mass spectrometry (ESIMS), and combustion analysis. The IR spectra of $\mathbf{4 a - 4 i}$ and $\mathbf{5 a}-\mathbf{5 h}$ exhibited the ring and the exocyclic $\mathrm{C}=\mathrm{O}$ bands in the $1692-1713 \mathrm{~cm}^{-1}$ and $1651-1670 \mathrm{~cm}^{-1}$, respectively. The shifts observed in the amide bands when compared to that of $3\left(1636 \mathrm{~cm}^{-1}\right)$ and the presence of additional lactam bands provided definite proof for the aimed cyclization. Observation of $\mathrm{NH}$ signals assigned to the indole $\mathrm{NH}$ $(\delta=12.11-12.18 \mathrm{ppm})$ and amide $\mathrm{NH}(9.97-0.21 \mathrm{ppm})$ together with the absence of the $\mathrm{NH}_{2}$ resonance of the intermediate hydrazide $\mathbf{3}$ in the ${ }^{1} \mathrm{H}$ NMR spectra of $\mathbf{4}$ and $\mathbf{5}$, provided further evidence for the formation of new adducts. The $\mathrm{S}-\mathrm{CH}_{2}(\mathbf{4 a}-\mathbf{4 i})$ and $\mathrm{S}-\mathrm{CH}(\mathbf{5} \mathbf{a}-\mathbf{5 h})$ protons of the newly formed spiroalkane residue resonated at about 3.50-3.65 and 3.87-3.93 ppm, respectively. The $\mathrm{S}-\mathrm{CH}_{2}$ protons of $\mathbf{4 a}-\mathbf{4 i}$ appeared as singlets except for the methylene hydrogens of compound $\mathbf{4 g}$ which were observed as separate doublets with large coupling constants $(J=16.1 \mathrm{~Hz})$ due to the geminal coupling resulting from the chiral centers of the spirothiazolidinone ring. The ${ }^{1} \mathrm{H}$ NMR spectra of $\mathbf{5 a}-\mathbf{5 h}$ displayed the thiazolidinone $\mathrm{S}-\mathrm{CH}$ protons as quartets or broad/distorted singlets and doublets. Assignment of the indole protons was achieved on the basis of the values and coupling constants reported for the 2,3,5-trisubstituted indole ring [15, 16, $24,25]$. The carbon resonances were assigned by chemical shifts and comparison with previously reported ${ }^{13} \mathrm{C} N M R$ data for compounds having a similar backbone structure [15, $19,26] . \mathrm{CH}_{3}, \mathrm{CH}_{2}, \mathrm{CH}$, and $\mathrm{C}$ signals were assigned by 
Table 1 Antitubercular activity against M. tuberculosis $\mathrm{H} 37 \mathrm{Rv}$ ATCC 27294

\begin{tabular}{llllll}
\hline Compound & $\mathrm{R}$ & $\mathrm{R}^{1}$ & $\mathrm{R}^{2}$ & $\mathrm{R}^{3}$ & $\mathrm{MIC} / \mu \mathrm{M}$ \\
\hline $\mathbf{4 a}$ & - & - & - & - & 1000 \\
$\mathbf{4 b}$ & $\mathrm{H}$ & $\mathrm{H}$ & $\mathrm{H}$ & $\mathrm{H}$ & 16 \\
$\mathbf{4 c}$ & $\mathrm{CH}_{3}$ & $\mathrm{H}$ & $\mathrm{H}$ & $\mathrm{H}$ & 16 \\
$\mathbf{4 d}$ & $\mathrm{H}$ & $\mathrm{CH}_{3}$ & $\mathrm{H}$ & $\mathrm{H}$ & 31 \\
$\mathbf{4 e}$ & $\mathrm{H}$ & $\mathrm{C}_{2} \mathrm{H}_{5}$ & $\mathrm{H}$ & $\mathrm{H}$ & 250 \\
$\mathbf{4 f}$ & $\mathrm{H}$ & $\mathrm{C}_{3} \mathrm{H}_{7}$ & $\mathrm{H}$ & $\mathrm{H}$ & 31 \\
$\mathbf{4 g}$ & $\mathrm{CH}_{3}$ & $\mathrm{H}$ & $\mathrm{CH}_{3}$ & $\mathrm{CH}_{3}$ & 500 \\
$\mathbf{4 h}$ & $\mathrm{H}$ & $\mathrm{C}_{6} \mathrm{H}_{5}$ & $\mathrm{H}$ & $\mathrm{H}$ & 3.9 \\
$\mathbf{4 i}$ & $\mathrm{H}$ & $\left.\mathrm{C}_{2} \mathrm{CH}_{3}\right)_{3}$ & $\mathrm{H}$ & $\mathrm{H}$ & 16 \\
$\mathbf{5 a}$ & - & - & - & - & 250 \\
$\mathbf{5 b}$ & $\mathrm{H}$ & $\mathrm{H}$ & $\mathrm{H}$ & $\mathrm{H}$ & 250 \\
$\mathbf{5 c}$ & $\mathrm{CH}_{3}$ & $\mathrm{H}$ & $\mathrm{H}$ & $\mathrm{H}$ & 500 \\
$\mathbf{5 d}$ & $\mathrm{H}$ & $\mathrm{CH}_{3}$ & $\mathrm{H}$ & $\mathrm{H}$ & 1000 \\
$\mathbf{5 e}$ & $\mathrm{H}$ & $\mathrm{C}_{2} \mathrm{H}_{5}$ & $\mathrm{H}$ & $\mathrm{H}$ & 1000 \\
$\mathbf{5 f}$ & $\mathrm{H}$ & $\mathrm{C}_{3} \mathrm{H}_{7}$ & $\mathrm{H}$ & $\mathrm{H}$ & 1000 \\
$\mathbf{5 g}$ & $\mathrm{CH}_{3}$ & $\mathrm{H}$ & $\mathrm{CH}$ & $\mathrm{CH}$ & 1000 \\
$\mathbf{5 h}$ & $\mathrm{H}$ & $\mathrm{C}_{6} \mathrm{H}_{5}$ & $\mathrm{H}$ & $\mathrm{H}$ & 7.8 \\
Rifampicin & - & - & - & - & $<0.1 \mu \mathrm{g} / \mathrm{cm}^{3}$ \\
\hline
\end{tabular}

The experiment was performed twice and the same results were obtained

${ }^{a}$ MIC, the actual minimum inhibitory concentration required to inhibit the growth of $100 \%$ of organisms

APT and DEPT experiments. Observation of the typical C5 $(\delta=70.59-76.40 \mathrm{ppm})$ and $\mathrm{C}=\mathrm{O}(167.64-170.57 \mathrm{ppm})$ resonances of the spirothiazolidinone structure in the ${ }^{13} \mathrm{C}$ NMR spectra of $\mathbf{4}$ and $\mathbf{5}$, verified the formation of the expected spiro ring system.

Deprotonated $[\mathrm{M}-\mathrm{H}]^{-}$or protonated $[\mathrm{M}+\mathrm{H}]^{+}$ions observed in the ESI-MS confirmed the molecular weight of the compounds. Additional $[(\mathrm{M}-\mathrm{H})+2]^{-}$or $[(\mathrm{M}+\mathrm{H})+2]^{+}$ isotope peaks approximately one-third the intensity of the molecular ion peak resulting from the ${ }^{37} \mathrm{Cl}$ isotope further confirmed the new structures.

\section{Antitubercular activity}

The antitubercular activity of compounds $\mathbf{4 a}-\mathbf{4 i}$ and $\mathbf{5 a}-\mathbf{5 h}$ was tested in vitro against M. tuberculosis $\mathrm{H} 37 \mathrm{Rv}$ ATCC 27294 using the microdilution method. The lowest concentration of compound that inhibited $100 \%$ of mycobacterial growth in the culture was defined as the MIC. Rifampicin was used as the reference drug. Compounds were assayed using twofold dilutions starting at $1000 \mu \mathrm{M}$. As shown in Table 1, compounds $\mathbf{4} \mathbf{h}$ and $\mathbf{5 h}$, bearing a phenyl substituent at position 8 of the spiro ring, exhibited the highest anti-TB activity at concentrations of 3.9 and $7.8 \mu \mathrm{M}$, respectively. Most of the compounds in series $4(\mathbf{4 b}, \mathbf{4 c}, \mathbf{4 d}$, 4f, and 4i) displayed some activity on mycobacteria with MIC values of 16 and $31 \mu \mathrm{M}$.

Looking at the chemical structures of the active compounds, it can be observed that the presence of a methyl group at position 2 of the spirocyclic system (series 5) led to a significant reduction in antitubercular activity. Introduction of a bulky aromatic substituent $\left(\mathrm{C}_{6} \mathrm{H}_{5}\right)$ at position 8 of the ring, as in $\mathbf{4 h}$ and $\mathbf{5 h}$, enhanced the antitubercular activity.

\section{Antiviral activity}

Compounds $4 \mathbf{a}-\mathbf{4 i}$ and $\mathbf{5 a}-\mathbf{5 h}$ were evaluated against a variety of DNA and RNA viruses in cell culture, namely: herpes simplex virus type-1 (HSV-1) and type-2 (HSV-2), an acyclovir-resistant thymidine kinase-deficient $\left(\mathrm{TK}^{-}\right)$mutant of HSV-1, vaccinia virus, human adenovirus-2, human coronavirus, vesicular stomatitis virus, Coxsackie B4 virus, respiratory syncytial virus, parainfluenza-3 virus, reovirus, Sindbis virus, Punta Toro virus, yellow fever virus, and influenza A and influenza $B$ virus. The cytopathic effect reduction assays revealed that compounds $\mathbf{4 b}, \mathbf{4 c}, \mathbf{5 b}$, and $\mathbf{5 d}$ were moderately active against Punta Toro virus, yellow fever virus or Sindbis virus in Vero cells (Table 2). The antiviral $\mathrm{EC}_{50}$ values were in the range of $1.9-12 \mu \mathrm{M}$ and the selectivity index (SI: ratio of cytotoxic to antivirally effective concentration) was above 10 in some cases (see values between brackets in Table 2). The most potent effect was seen with compound 5d that is methylated at positions 2 and 8 of the spirothiazolidinone system. Of note, no antiviral activity was obtained for the analogues carrying a spiro-fused cyclopentane ring instead of cyclohexane (i.e., 4a and 5a) or a bulkier group than methyl on the cyclohexane residue. Introduction of a methyl group at position 2 of the ring system (e.g., compare compounds $\mathbf{4 b}$ and $\mathbf{5 b}$ ) seemed to have a slightly positive effect on antiviral activity.

The test compounds did not exhibit activity against any of the other DNA- or RNA-viruses tested. Nevertheless, this broad antiviral testing allowed to determine the compounds' cytotoxic activity in different mammalian cell lines (Table 3). In general, the compounds endowed with antiviral activity $(\mathbf{4 b}, \mathbf{4 c}, \mathbf{5 b}$, and $\mathbf{5 d})$ tended to be less cytotoxic than the inactive derivatives.

\section{Antibacterial and antifungal activity}

The broad antibacterial and antifungal activity of compounds $4 \mathrm{a}-4 \mathrm{i}$ and $5 \mathrm{a}-5 \mathrm{~h}$ was further assessed using Pseudomonas aeruginosa ATCC 27853, Escherichia coli ATCC 25922, Klebsiella pneumoniae ATCC 4352, Proteus mirabilis ATCC 14153, Staphylococcus aureus ATCC 29213, Staphylococcus epidermidis ATCC 12228, Enterococcus faecalis ATCC 29212, Candida albicans ATCC 10231, Candida parapsilosis ATCC 22019, and Candida tropicalis ATCC 
Table 2 Antiviral activity in Vero ${ }^{\mathrm{a}}$ cell cultures infected with diverse RNA viruses

\begin{tabular}{|c|c|c|c|c|c|c|c|c|c|c|c|}
\hline \multirow[t]{3}{*}{ Compound } & \multirow[t]{3}{*}{$R$} & \multirow[t]{3}{*}{$R^{1}$} & \multirow[t]{3}{*}{$R^{2}$} & \multirow[t]{3}{*}{$R^{3}$} & \multicolumn{7}{|c|}{ Antiviral assays in Vero cells } \\
\hline & & & & & \multicolumn{6}{|c|}{ Antiviral $\mathrm{EC}_{50}^{\mathrm{b}}$ value $/ \mu \mathrm{M}$} & \multirow[t]{2}{*}{$\mathrm{MCC}^{\mathrm{c}} / \mu \mathrm{M}$} \\
\hline & & & & & $\begin{array}{l}\text { Parain- } \\
\text { fluenza-3 } \\
\text { virus }\end{array}$ & Reovirus-1 & $\begin{array}{l}\text { Sindbis } \\
\text { virus }\end{array}$ & $\begin{array}{l}\text { Cox- } \\
\text { sackie B4 } \\
\text { virus }\end{array}$ & $\begin{array}{l}\text { Punta Toro } \\
\text { virus }\end{array}$ & $\begin{array}{l}\text { Yellow } \\
\text { Fever virus }\end{array}$ & \\
\hline $4 \mathbf{a}$ & - & - & - & - & $>100$ & $>100$ & $>100$ & $>100$ & $>100$ & $>100$ & $>100$ \\
\hline $4 b$ & $\mathrm{H}$ & $\mathrm{H}$ & $\mathrm{H}$ & $\mathrm{H}$ & $>100$ & $>100$ & $>100$ & $>100$ & $11[9]$ & $>100$ & 100 \\
\hline $4 c$ & $\mathrm{CH}_{3}$ & $\mathrm{H}$ & $\mathrm{H}$ & $\mathrm{H}$ & $>100$ & $>100$ & $>100$ & $>100$ & $12[8]$ & $>100$ & 100 \\
\hline $4 d$ & $\mathrm{H}$ & $\mathrm{CH}_{3}$ & $\mathrm{H}$ & $\mathrm{H}$ & $>100$ & $>100$ & $>100$ & $>100$ & $>100$ & $>100$ & 20 \\
\hline $4 e$ & $\mathrm{H}$ & $\mathrm{C}_{2} \mathrm{H}_{5}$ & $\mathrm{H}$ & $\mathrm{H}$ & $>100$ & $>100$ & $>100$ & $>100$ & $>100$ & $>100$ & 20 \\
\hline $4 f$ & $\mathrm{H}$ & $\mathrm{C}_{3} \mathrm{H}_{7}$ & $\mathrm{H}$ & $\mathrm{H}$ & $>100$ & $>100$ & $>100$ & $>100$ & $>100$ & $>100$ & 20 \\
\hline $4 g$ & $\mathrm{CH}_{3}$ & $\mathrm{H}$ & $\mathrm{CH}_{3}$ & $\mathrm{CH}_{3}$ & $>100$ & $>100$ & $>100$ & $>100$ & $>100$ & $>100$ & 20 \\
\hline $4 h$ & $\mathrm{H}$ & $\mathrm{C}_{6} \mathrm{H}_{5}$ & $\mathrm{H}$ & $\mathrm{H}$ & $>100$ & $>100$ & $>100$ & $>100$ & $>100$ & $>100$ & 20 \\
\hline $4 i$ & $\mathrm{H}$ & $\mathrm{C}\left(\mathrm{CH}_{3}\right)_{3}$ & $\mathrm{H}$ & $\mathrm{H}$ & $>100$ & $>100$ & $>100$ & $>100$ & $>100$ & $>100$ & 20 \\
\hline $5 a$ & - & - & - & - & $>100$ & $>100$ & $>100$ & $>100$ & $>100$ & $>100$ & $>100$ \\
\hline $5 b$ & $\mathrm{H}$ & $\mathrm{H}$ & $\mathrm{H}$ & $\mathrm{H}$ & $>100$ & $>100$ & $>100$ & $>100$ & $>100$ & $5.6[>18]$ & $>100$ \\
\hline $5 c$ & $\mathrm{CH}_{3}$ & $\mathrm{H}$ & $\mathrm{H}$ & $\mathrm{H}$ & $>100$ & $>100$ & $>100$ & $>100$ & $>100$ & $>100$ & $>100$ \\
\hline $5 d$ & $\mathrm{H}$ & $\mathrm{CH}_{3}$ & $\mathrm{H}$ & $\mathrm{H}$ & $>100$ & $>100$ & $8.9[\geq 2]$ & $>100$ & $>100$ & $1.9[\geq 11]$ & $\geq 20$ \\
\hline $5 e$ & $\mathrm{H}$ & $\mathrm{C}_{2} \mathrm{H}_{5}$ & $\mathrm{H}$ & $\mathrm{H}$ & $>100$ & $>100$ & $>100$ & $>100$ & $>100$ & $>100$ & 20 \\
\hline $5 f$ & $\mathrm{H}$ & $\mathrm{C}_{3} \mathrm{H}_{7}$ & $\mathrm{H}$ & $\mathrm{H}$ & $>100$ & $>100$ & $>100$ & $>100$ & $>100$ & $>100$ & 20 \\
\hline $5 g$ & $\mathrm{CH}_{3}$ & $\mathrm{H}$ & $\mathrm{CH}_{3}$ & $\mathrm{CH}_{3}$ & $>100$ & $>100$ & $>100$ & $>100$ & $>100$ & $>100$ & $>100$ \\
\hline $5 h$ & $\mathrm{H}$ & $\mathrm{C}_{6} \mathrm{H}_{5}$ & $\mathrm{H}$ & $\mathrm{H}$ & $>100$ & $>100$ & $>100$ & $>100$ & $>100$ & $>100$ & 20 \\
\hline DS $-10,000^{\mathrm{d}}$ & - & - & - & - & $>100$ & $>100$ & 0.8 & 34 & 4.0 & 15 & $>100$ \\
\hline Ribavirin & - & - & - & - & 111 & 111 & 8.9 & $>250$ & 111 & $>250$ & $>250$ \\
\hline Mycophenolic acid & - & - & - & - & 0.4 & 1.5 & 0.4 & $>100$ & 1.7 & 0.8 & $>100$ \\
\hline
\end{tabular}

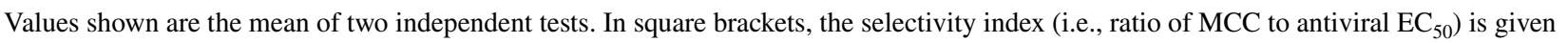
${ }^{a}$ Vero: African green monkey kidney cells

${ }^{\mathrm{b}} \mathrm{EC}_{50}: 50 \%$ effective concentration, producing $50 \%$ inhibition of virus-induced cytopathic effect, as determined by microscopy

${ }^{\mathrm{c}} \mathrm{MCC}$ : minimal cytotoxic concentration, or compound concentration causing minimal changes in cell morphology, as assessed by microscopy

${ }^{\mathrm{d}}$ DS-10,000: dextran sulfate with MW 10,000; for this compound, data are expressed in $\mu \mathrm{g}$ per $\mathrm{cm}^{3}$

750. The compounds were assayed using twofold dilutions from 2500 to $156 \mu \mathrm{M}$. Neither of the test compounds produced antimicrobial activity below this concentration range.

\section{Conclusion}

In the search for effective antimicrobial agents, we achieved the synthesis of novel spirothiazolidinone derivatives $\mathbf{4 a}-\mathbf{4 i}$ 5a-5h with the 5-chloro-3-phenyl-1H-indole scaffold. Structures of the new compounds were characterized and confirmed by spectrometric methods (IR, ${ }^{1} \mathrm{H}$ NMR, ${ }^{13} \mathrm{C}$ NMR, and ESI-MS) and elemental analysis. Compounds $4 \mathbf{a}-4 \mathbf{i}$ and $5 \mathbf{a}-\mathbf{5 h}$ were evaluated for in vitro antitubercular, antiviral, antibacterial, and antifungal activity against various viral, bacterial, and fungal strains. Compounds $\mathbf{4 h}$ and $\mathbf{5 h}$, bearing a bulky phenyl group at position 8 of the spiro ring, displayed appreciable anti-TB activity against $M$. tuberculosis H37Rv ATCC 27294 with MIC values of 3.9 and $7.8 \mu \mathrm{M}$, respectively. Compounds $\mathbf{4 b}, \mathbf{4 c}, \mathbf{5 b}$, and $\mathbf{5 d}$ exhibited inhibitory effect on the replication of Punta Toro virus, yellow fever virus or Sindbis virus in Vero cells. The antiviral $\mathrm{EC}_{50}$ values were in the range of $1.9-12 \mu \mathrm{M}$ and the selectivity index (SI: ratio of cytotoxic to antivirally effective concentration) was above 10 in some cases. The most potent effect was seen with compound $\mathbf{5 d}$ that is methylated at positions 2 and 8 of the spirothiazolidinone system. Neither of the indole-spirothiazolidinone compounds showed activity against any of the bacterial or fungal strains tested, at concentrations below $2500-156 \mu \mathrm{M}$.

\section{Experimental}

All purchased solvents and chemicals were of analytical grade and used as received. Melting points were determined in open capillary tubes with a Buchi B-540 melting point apparatus. Microanalyses were performed on a Thermo 
Table 3 Cytotoxic activity in diverse mammalian cell lines ${ }^{\mathrm{a}}$

\begin{tabular}{llllll}
\hline Compound & \multicolumn{2}{l}{$\mathrm{MCC} / \mu \mathrm{M}$} & $\mathrm{CC}_{50}^{\mathrm{c}} / \mu \mathrm{M}$ \\
\cline { 2 - 4 } & $\mathrm{HEL}$ & $\mathrm{HeLa}$ & Vero & $\mathrm{MDCK}$ & $\mathrm{MDCK}$ \\
\hline $\mathbf{4 a}$ & $>100$ & $>100$ & $>100$ & $>100$ & $>100$ \\
$\mathbf{4 b}$ & 100 & 100 & 100 & $\geq 20$ & 63 \\
$\mathbf{4 c}$ & 100 & 100 & 100 & $\geq 20$ & 64 \\
$\mathbf{4 d}$ & 20 & 20 & 20 & 4 & 2.3 \\
$\mathbf{4 e}$ & 20 & 20 & 20 & 4 & 9.9 \\
$\mathbf{4 f}$ & 20 & 20 & 20 & 4 & 2.4 \\
$\mathbf{4 g}$ & 20 & 20 & 20 & 4 & 20 \\
$\mathbf{4 h}$ & 20 & 20 & 20 & 4 & 2.3 \\
$\mathbf{4 i}$ & 20 & 20 & 20 & 4 & 2.3 \\
$\mathbf{5 a}$ & $>100$ & $>100$ & $>100$ & $\geq 100$ & $>100$ \\
$\mathbf{5 b}$ & 100 & $\geq 100$ & $>100$ & 100 & $>100$ \\
$\mathbf{5 c}$ & $>100$ & $>100$ & $>100$ & $>100$ & $>100$ \\
$\mathbf{5 d}$ & 20 & 20 & $\geq 20$ & $\geq 20$ & $>100$ \\
$\mathbf{5 e}$ & 20 & 20 & 20 & 4 & 18 \\
$\mathbf{5 f}$ & 20 & 20 & 20 & 4 & 2.3 \\
5g & $\geq 100$ & 100 & $>100$ & 100 & $>100$ \\
$\mathbf{5 h}$ & 20 & 20 & 20 & 4 & 2.3 \\
DS-10,000 & $\mathrm{ND}$ & $>100$ & $>100$ & $\mathrm{ND}$ & $\mathrm{ND}$ \\
Ribavirin & $\mathrm{ND}$ & $\geq 250$ & $>250$ & $>100$ & $>100$ \\
Ganciclovir & $>100$ & $\mathrm{ND}$ & $\mathrm{ND}$ & $\mathrm{ND}$ & $\mathrm{ND}$ \\
Brivudin & $>250$ & $\mathrm{ND}$ & $\mathrm{ND}$ & $\mathrm{ND}$ & $\mathrm{ND}$ \\
\hline
\end{tabular}

$N D$ not done

${ }^{a} H E L$ human embryonic lung fibroblast cells, HeLa human cervix carcinoma cells, Vero African green monkey kidney cells, MDCK Madin-Darby canine kidney cells

${ }^{\mathrm{b}}$ MCC minimal cytotoxic concentration, or compound concentration causing minimal changes in cell morphology, as assessed by microscopy

${ }^{\mathrm{c}} \mathrm{CC}_{50} 50 \%$ cytotoxic concentration, assessed by the spectroscopic MTS cell viability assay

${ }^{\mathrm{d}} D S-10,000$ dextran sulfate with MW 10,000; for this compound, data are expressed in $\mu \mathrm{g}$ per $\mathrm{cm}^{3}$

Finnigan Flash EA 1112 elemental analyzer and their results were found to be in good agreement $( \pm 0.3 \%)$ with the calculated values. IR spectra were recorded in $\mathrm{KBr}$ discs ( $\bar{v} /$ $\mathrm{cm}^{-1}$ ) on a Shimadzu IRAffinity-1 FTIR spectrophotometer. ${ }^{1} \mathrm{H}$ NMR (DMSO- $d_{6}$ ), ${ }^{13} \mathrm{C}$ NMR (APT, DMSO- $d_{6}$ ) were run

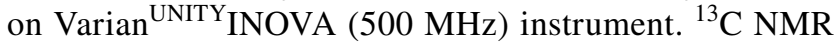
(DEPT, DMSO- $d_{6}$ ) were run Bruker ARX (300 MHz). Chemical shifts are reported as $\delta$ (ppm) relative to TMS as internal standard and coupling constants $(J)$ are given in hertz $(\mathrm{Hz})$. MS $(\mathrm{ESI} \pm)$ were determined on a Finnigan LCQ Advantage Max mass spectrometer (*: broad/distorted, ind.:indole, ar.:aromatic, sp.: spirodecane).

Ethyl 2-[2-(4-chlorophenyl)hydrazinylidene]-3-phenylpropanoate (1) [21] To a solution of 4-chloroaniline (0.02 mol) in $10 \mathrm{~cm}^{3}$ ethanol, $10 \mathrm{~cm}^{3}$ water, and $6 \mathrm{~cm}^{3}$ conc. $\mathrm{HCl}$,
$30 \mathrm{~cm}^{3}$ aqueous $\mathrm{NaNO}_{2}$ solution (7\%) was added dropwise at $0{ }^{\circ} \mathrm{C}$ with stirring. The resulting solution of diazonium salt was poured into a cooled $\left(0{ }^{\circ} \mathrm{C}\right)$ mixture of ethyl 2-benzyl-3-oxobutanoate $(0.02 \mathrm{~mol}), 10 \mathrm{~cm}^{3}$ ethanol, $10 \mathrm{~cm}^{3}$ water, and $5.4 \mathrm{~g} \mathrm{KOH}$ while stirring. The resulting mixture was refrigerated overnight. The red oily solid residue thus obtained was separated, washed with water, and used without further purification. Yield: $72.4 \%$; m.p.: $90-93{ }^{\circ} \mathrm{C}$ (Ref. [21] $87-93^{\circ} \mathrm{C}$ ).

Ethyl 5-chloro-3-phenyl-1H-indole-2-carboxylate (2) [22] A solution of $1(0.02 \mathrm{~mol})$ in $20 \mathrm{~cm}^{3}$ conc. $\mathrm{HCl}$ was heated under reflux on a water bath $\left(70-80{ }^{\circ} \mathrm{C}\right)$ for $4 \mathrm{~h}$. The crude product was filtered off, washed with water until tested neutral to litmus and used without further purification. Yield: 67.8\%; m.p.: $160-162{ }^{\circ} \mathrm{C}$ (Ref. [22] $158-160{ }^{\circ} \mathrm{C}$ ).

5-Chloro-3-phenyl - $1 \mathrm{H}$-indole-2-carbohydrazide (3) [23] A mixture of $2(0.02 \mathrm{~mol}), 20 \mathrm{~cm}^{3}$ ethanol, and $8 \mathrm{~cm}^{3}$ $\mathrm{H}_{2} \mathrm{NNH}_{2} \cdot \mathrm{H}_{2} \mathrm{O}(98 \%)$ was heated under reflux on a water bath $\left(70-80{ }^{\circ} \mathrm{C}\right.$ ) for $6 \mathrm{~h}$. The resulting brown solid was filtered off and recrystallized from ethanol. Yield: 53.7\%; m.p.: 227-230 ${ }^{\circ} \mathrm{C}$ (Ref. [23] 234-236 ${ }^{\circ} \mathrm{C}$ ).

\section{General procedure for the synthesis of 5-chloro-3-phenyl- $N$ - $(2,7,8,9-$ substituted/ nonsubstituted-3-oxo-1-thia-4-azaspiro[4.4]nonan/ [4.5]decan-4-yl)-1H-indole-2-carboxamides $4 a-4 i$, $5 a-5 h$}

A mixture of $\mathbf{3}(0.0025 \mathrm{~mol})$, an appropriate cyclohexanone/cyclopentanone $(0.003 \mathrm{~mol})$, and mercaptoacetic acid or 2-mercaptopropionic acid $(0.01 \mathrm{~mol})$ in $20 \mathrm{~cm}^{3}$ dry toluene was heated to reflux with a heating mantle for 5-6 h using a Dean-Stark water separator. Excess toluene was evaporated in vacuo. The resulting residue was treated with saturated $\mathrm{NaHCO}_{3}$ solution until $\mathrm{CO}_{2}$ evolution ceased and was allowed to stand overnight or in some cases refrigerated until solidification. The solid thus obtained was washed with water, dried, and recrystallized from ethanol or ethyl acetate.

5-Chloro- $N$-(3-oxo-1-thia-4-azaspiro[4.4]nonan-4-yl)-3-phenyl- $1 \mathrm{H}$-indole-2-carboxamide $\left(4 \mathrm{a}, \mathrm{C}_{22} \mathrm{H}_{20} \mathrm{CIN}_{3} \mathrm{O}_{2} \mathrm{~S}\right)$ White crystals; yield: $87.7 \%$; m.p.: $297-300{ }^{\circ} \mathrm{C} ; R_{f}=0.46$ (EtOAc/ $\mathrm{CHCl}_{3}$ 1:1); IR (KBr): $\bar{\nu}=3277(\mathrm{~N}-\mathrm{H}), 1709,1651(\mathrm{C}=\mathrm{O})$ $\mathrm{cm}^{-1} ;{ }^{1} \mathrm{H}$ NMR (DMSO- $\left.d_{6}, 500 \mathrm{MHz}\right): \delta=1.58-1.69(4 \mathrm{H}$, m, $\mathrm{CH}_{2}$-sp.), 1.72-1.74 (2H, m, $\mathrm{CH}_{2}$-sp.), 1.94 (2H, s*, $\mathrm{CH}_{2}$-sp.), 3.65 (2H, s, S-CH${ }_{2}$-sp.), $7.29(1 \mathrm{H}, \mathrm{dd}, J=8.5$, $2.0 \mathrm{~Hz}, \mathrm{H6}$-ind.), $7.37\left(1 \mathrm{H}, \mathrm{tt}, J=7.3,1.5 \mathrm{~Hz}, 3-\mathrm{C}_{6} \mathrm{H}_{5}(\mathrm{H} 4)\right.$ ind.), $7.45\left(2 \mathrm{H}, \mathrm{t}, J=7.3 \mathrm{~Hz}, 3-\mathrm{C}_{6} \mathrm{H}_{5}(\mathrm{H} 3, \mathrm{H} 5)\right.$-ind.), $7.51-$ 7.54 (4H, m, H4, H7, 3- $\mathrm{C}_{6} \mathrm{H}_{5}(\mathrm{H} 2, \mathrm{H} 6)$-ind.), $10.10(1 \mathrm{H}, \mathrm{s}$, $\mathrm{CONH}), 12.13(1 \mathrm{H}, \mathrm{s}, \mathrm{NH}) \mathrm{ppm} ;{ }^{13} \mathrm{C}$ NMR (APT, DMSO$\left.d_{6}, 125 \mathrm{MHz}\right): \delta=22.40,29.05,37.91\left(\mathrm{CH}_{2}\right.$-sp. $), 76.40$ 
(C5-sp.), 114.62, 114.67 (CH-ar.), 118.64 (C-ar.), 119.32, 119.41 (CH-ar.), 124.69 (CH-ar.), 125.50 (C-ar.), 127.75 (CH-ar.), 127.81 (C-ar.), 128.03 (C-ar.), 128.79 (CH-ar.), 130.44 (CH-ar.), 133.27 (C-ar.), 134.65 (C-ar.), 162.08 (CONH), 167.87 (CO-sp.) ppm; MS (ESI-): $m / z(\%)=424.5$ $\left([\mathrm{M}-\mathrm{H}]^{-}, 100\right), 426.3\left([(\mathrm{M}-\mathrm{H})+2]^{-}, 36.5\right)$.

5-Chloro- $N$-(3-oxo-1-thia-4-azaspiro[4.5]decan-4-yl)-3-phenyl-1H-indole-2-carboxamide $\left(4 \mathrm{~b}, \mathrm{C}_{23} \mathrm{H}_{22} \mathrm{CIN}_{3} \mathrm{O}_{2} \mathrm{~S}\right.$ ) White crystals; yield: $76.9 \%$; m.p.: $229-232{ }^{\circ} \mathrm{C} ; R_{f}=0.48$ (EtOAc/ $\left.\mathrm{CHCl}_{3} 1: 1\right)$; IR (KBr): $\bar{v}=3279(\mathrm{~N}-\mathrm{H}), 1709,1651(\mathrm{C}=\mathrm{O})$ $\mathrm{cm}^{-1} ;{ }^{1} \mathrm{H}$ NMR (DMSO- $\left.d_{6}, 500 \mathrm{MHz}\right): \delta=1.01-1.11(2 \mathrm{H}$, m, $\mathrm{CH}_{2}$-sp.), 1.35-1.42 (2H, m, $\mathrm{CH}_{2}$-sp.), $1.56\left(1 \mathrm{H}, \mathrm{d}^{*}\right.$, $J=13.2 \mathrm{~Hz}, \mathrm{CH}_{2}$-sp.), $1.64-1.72\left(5 \mathrm{H}, \mathrm{m}, \mathrm{CH}_{2}\right.$-sp.), 3.58 (2H, s, S-CH $-\mathrm{CH}_{2}$-sp.), 7.30 (1H, dd, $J=8.8,2.4 \mathrm{~Hz}, \mathrm{H} 6$-ind.), $7.37\left(1 \mathrm{H}, \mathrm{tt}, J=7.3,1.5 \mathrm{~Hz}, 3-\mathrm{C}_{6} \mathrm{H}_{5}(\mathrm{H} 4)-\mathrm{ind}.\right), 7.46(2 \mathrm{H}, \mathrm{t}$, $J=7.6 \mathrm{~Hz}, 3-\mathrm{C}_{6} \mathrm{H}_{5}(\mathrm{H} 3, \mathrm{H} 5)$-ind.), 7.51-7.56 (4H, m, H4, H7, $3-\mathrm{C}_{6} \mathrm{H}_{5}(\mathrm{H} 2, \mathrm{H} 6)$-ind.), 10.09 (1H, s, CONH), $12.14(1 \mathrm{H}, \mathrm{s}$, $\mathrm{NH}$ ) ppm; ${ }^{13} \mathrm{C}$ NMR (APT, DMSO- $d_{6}, 125 \mathrm{MHz}$ ): $\delta=23.33$, 24.31, 28.29, 37.10 ( $\mathrm{CH}_{2}$-sp.), 72.63 (C5-sp.), 114.56, 114.64 (CH-ar.), 118.50 (C-ar.), 119.31, 119.39 (CH-ar.), 124.54, 124.66 (CH-ar.), 125.47 (C-ar.), 127.41 (CH-ar.), 127.82 (C-ar.), 128.23 (C-ar.), 128.90 (CH-ar.), 130.51 (CHar.), 133.38 (C-ar.), 134.62 (C-ar.), 162.14 (CO-NH), 167.73 (CO-sp.) ppm.

5-Chloro- $N$-(7-methyl-3-oxo-1-thia-4-azaspiro[4.5]decan-4-yl)-3-phenyl- $1 \mathrm{H}$-indole-2-carboxamide (4c, $\mathrm{C}_{24} \mathrm{H}_{24} \mathrm{CIN}_{3} \mathrm{O}_{2} \mathrm{~S}$ ) White powder; yield: 83.4\%; m.p.: 268 $270{ }^{\circ} \mathrm{C} ; R_{f}=0.52\left(\mathrm{EtOAc} / \mathrm{CHCl}_{3} 1: 1\right)$; IR (KBr): $\bar{v}=3231$ $(\mathrm{N}-\mathrm{H}), 1711,1651(\mathrm{C}=\mathrm{O}) \mathrm{cm}^{-1} ;{ }^{1} \mathrm{H}$ NMR (DMSO- $d_{6}$, $500 \mathrm{MHz}): \delta=0.76-0.85\left(1 \mathrm{H}, \mathrm{m}, \mathrm{CH} / \mathrm{CH}_{2}\right.$-sp.), $0.87(3 \mathrm{H}$, d, $J=6.4 \mathrm{~Hz}, 7-\mathrm{CH}_{3}$-sp.), $1.24-1.70\left(8 \mathrm{H}, \mathrm{m}, \mathrm{CH} / \mathrm{CH}_{2}\right.$-sp.), $3.58\left(2 \mathrm{H}, \mathrm{s}, \mathrm{S}-\mathrm{CH}_{2}\right.$-sp.), $7.29(1 \mathrm{H}, \mathrm{dd}, J=8.8,2.0 \mathrm{~Hz}$, H6-ind.), $7.37\left(1 \mathrm{H}, \mathrm{tt}, J=7.3,1.5 \mathrm{~Hz}, 3-\mathrm{C}_{6} \mathrm{H}_{5}(\mathrm{H} 4)\right.$-ind.), $7.45\left(2 \mathrm{H}, \mathrm{t}, J=7.3 \mathrm{~Hz}, 3-\mathrm{C}_{6} \mathrm{H}_{5}(\mathrm{H} 3, \mathrm{H} 5)-\right.$ ind. $), 7.49(1 \mathrm{H}, \mathrm{d}$, $J=2.0 \mathrm{~Hz}, \mathrm{H} 4$-ind.), 7.52-7.54 (3H, m, H7, 3- $\mathrm{C}_{6} \mathrm{H}_{5}(\mathrm{H} 2, \mathrm{H} 6)$ ind.), $10.06(1 \mathrm{H}, \mathrm{s}, \mathrm{CONH}), 12.14(1 \mathrm{H}, \mathrm{s}, \mathrm{NH}) \mathrm{ppm} ;{ }^{13} \mathrm{C}$ NMR (APT, DMSO- $\left.d_{6}, 125 \mathrm{MHz}\right): \delta=22.42\left(7-\mathrm{CH}_{3}\right.$-sp.), 22.88, 28.36 ( $\mathrm{CH}_{2}$-sp.), 29.97 (C7-sp.), 32.98, 36.54, 45.20 ( $\mathrm{CH}_{2}$-sp.), 72.59 (C5-sp.), 114.56, 114.63 (CH-ar.), 118.50 (C-ar.), 119.28, 119.37 (CH-ar.), 124.54 (CH-ar.), 125.45 (C-ar.), 127.42 (CH-ar.), 127.85 (C-ar.), 128.25 (C-ar.), 128.91 (CH-ar.), 130.51 (CH-ar.), 133.43 (C-ar.), 134.62 (C-ar.), 162.18 (CO-NH), 167.66 (CO-sp.) ppm.

5-Chloro- $N$-(8-methyl-3-oxo-1-thia-4-azaspiro[4.5]decan-4-yl)-3-phenyl-1H-indole-2-carboxamide (4d, $\mathrm{C}_{24} \mathrm{H}_{24} \mathrm{CIN}_{3} \mathrm{O}_{2} \mathrm{~S}$ ) White powder; yield: $91.2 \%$; m.p.: 292 $293{ }^{\circ} \mathrm{C} ; R_{f}=0.51\left(\mathrm{EtOAc} / \mathrm{CHCl}_{3} 1: 1\right)$; IR (KBr): $\bar{v}=3285$ $(\mathrm{N}-\mathrm{H}), 1709,1651(\mathrm{C}=\mathrm{O}) \mathrm{cm}^{-1} ;{ }^{1} \mathrm{H}$ NMR (DMSO- $d_{6}$, $500 \mathrm{MHz}): \delta=0.89,0.95\left(3 \mathrm{H}, 2 \mathrm{~d}, J=6.4 \mathrm{~Hz}, 8-\mathrm{CH}_{3}\right.$-sp.), 1.08-1.32 (3H, m, CH/CH ${ }_{2}$-sp.), 1.64-1.75 (6H, m, CH/
$\mathrm{CH}_{2}$-sp.), 3.57, 3.58 (2H, $2 \mathrm{~s}, \mathrm{~S}-\mathrm{CH}_{2}$-sp.), 7.29, $7.30(1 \mathrm{H}$, $2 \mathrm{dd}, J=8.5,2.4 \mathrm{~Hz}, \mathrm{H6}$-ind.), $7.36(1 \mathrm{H}, \mathrm{tt}, J=8.0,1.5 \mathrm{~Hz}$, $3-\mathrm{C}_{6} \mathrm{H}_{5}(\mathrm{H} 4)$-ind.), $7.46\left(2 \mathrm{H}, \mathrm{t}, J=7.8 \mathrm{~Hz}, 3-\mathrm{C}_{6} \mathrm{H}_{5}(\mathrm{H} 3, \mathrm{H} 5)\right.$ ind.), 7.51-7.55 (4H, m, H4, H7, 3- $\mathrm{C}_{6} \mathrm{H}_{5}(\mathrm{H} 2, \mathrm{H} 6)$-ind.), $10.05,10.06(1 \mathrm{H}, 2 \mathrm{~s}, \mathrm{CONH}), 12.11,12.12(1 \mathrm{H}, 2 \mathrm{~s}, \mathrm{NH})$ ppm; ${ }^{13} \mathrm{C}$ NMR (proton decoupled and DEPT, DMSO$\left.d_{6}, 75 \mathrm{MHz}\right): \delta=22.23\left(8-\mathrm{CH}_{3}\right.$-sp.), $28.19\left(\mathrm{CH}_{2}\right.$-sp.), 30.70 (C8-sp.), 31.73, 36.76 ( $\mathrm{CH}_{2}$-sp.), 72.39 (C5-sp.), 114.50 (CH-ar.), 118.43 (C-ar.), 119.25 (CH-ar.), 124.52 (CH-ar.), 125.38 (C-ar.), 127.26 (CH-ar.), 127.73 (C-ar.), 128.06 (C-ar.), 128.76 (CH-ar.), 130.37 (CH-ar.), 133.26 (C-ar.), 134.53 (C-ar.), 162.00 (CO-NH), 167.69 (CO-sp.) ppm; MS (ESI-): $\mathrm{m} / \mathrm{z}(\%)=452.6\left([\mathrm{M}-\mathrm{H}]^{-}, 100\right), 454.4$ $\left([(\mathrm{M}-\mathrm{H})+2]^{-}, 34.3\right)$.

5-Chloro-N-(8-ethyl-3-oxo-1-thia-4-azaspiro[4.5]decan-4-yl)-3-phenyl-1H-indole-2-carboxamide (4e, $\mathrm{C}_{25} \mathrm{H}_{26} \mathrm{ClN}_{3} \mathrm{O}_{2} \mathrm{~S}$ ) White flakes; yield: $88.2 \%$; m.p.: 236$237{ }^{\circ} \mathrm{C} ; R_{f}=0.53\left(\mathrm{EtOAc} \mathrm{CHCl}_{3} 1: 1\right)$; IR (KBr): $\bar{\nu}=3283$ $(\mathrm{N}-\mathrm{H}), 1713,1651(\mathrm{C}=\mathrm{OC}=\mathrm{O}) \mathrm{cm}^{-1} ;{ }^{1} \mathrm{H}$ NMR $\left(\mathrm{DMSO}-d_{6}\right.$, $500 \mathrm{MHz}): \delta=0.89$ (3H, t, $J=7.3 \mathrm{~Hz}, 8-\mathrm{CH}_{2} \mathrm{CH}_{3}$-sp.), 1.04 $1.35\left(5 \mathrm{H}, \mathrm{m}, \mathrm{CH} / \mathrm{CH}_{2}\right.$-sp., 8- $\mathrm{C}_{2} \mathrm{CH}_{3}$-sp.), $1.62-1.96(6 \mathrm{H}$, $\mathrm{m}, \mathrm{CH} / \mathrm{CH}_{2}$-sp.), 3.58, $3.59\left(2 \mathrm{H}, 2 \mathrm{~s}, \mathrm{~S}-\mathrm{CH}_{2}\right.$-sp.), $7.30(1 \mathrm{H}$, dd, $J=8.8,2.0 \mathrm{~Hz}, \mathrm{H} 6$-ind.), $7.36(1 \mathrm{H}, \mathrm{tt}, J=7.3,1.5 \mathrm{~Hz}$, $3-\mathrm{C}_{6} \mathrm{H}_{5}(\mathrm{H} 4)$-ind.), 7.45 (2H, t, $J=7.3 \mathrm{~Hz}, 3-\mathrm{C}_{6} \mathrm{H}_{5}(\mathrm{H} 3, \mathrm{H} 5)$ ind.), 7.51-7.56 (4H, m, H4, H7, 3- $\mathrm{C}_{6} \mathrm{H}_{5}(\mathrm{H} 2, \mathrm{H} 6)$-ind.), $10.09,10.10(1 \mathrm{H}, 2 \mathrm{~s}, \mathrm{CONH}), 12.13(1 \mathrm{H}, \mathrm{s}, \mathrm{NH}) \mathrm{ppm} ;{ }^{13} \mathrm{C}$ NMR (APT, DMSO- $\left.d_{6}, 125 \mathrm{MHz}\right): \delta=11.94\left(8-\mathrm{CH}_{2} \mathrm{CH}_{3}-\right.$ sp.), 28.29, 28.35, 29.22, 29.41, $36.84\left(\mathrm{CH}_{2}\right.$-sp., 8- $\mathrm{CH}_{2} \mathrm{CH}_{3}$ sp.), 37.45 (C8-sp.), 72.75 (C5-sp.), 114.55, 114.63 (CHar.), 118.48 (C-ar.), 119.29, 119.38 (CH-ar.), 124.64 (CH-ar.), 125.47 (C-ar.), 127.40 (CH-ar.), 127.81 (C-ar.), 128.24 (C-ar.), 128.87 (CH-ar.), 130.48 (CH-ar.), 133.38 (C-ar.), 134.60 (C-ar.), 162.11 (CO-NH), 167.78 (CO-sp.) ppm; MS (ESI-): $m / z(\%)=466.8\left([\mathrm{M}-\mathrm{H}]^{-}, 100\right), 468.5$ $\left([(\mathrm{M}-\mathrm{H})+2]^{-}, 30.9\right)$.

5-Chloro-3-phenyl- $\mathrm{N}$-(8-propyl-3-oxo-1-thia-4-azaspiro[4.5]decan-4-yl)-1 H-indole-2-carboxamide (4f, $\left.\mathrm{C}_{26} \mathrm{H}_{28} \mathrm{CIN}_{3} \mathrm{O}_{2} \mathrm{~S}\right)$ Beige powder; yield: 75.7\%; m.p.: 233$236{ }^{\circ} \mathrm{C} ; R_{f}=0.55\left(\mathrm{EtOAc} / \mathrm{CHCl}_{3} 1: 1\right)$; IR (KBr): $\bar{v}=3291$, $3217(\mathrm{~N}-\mathrm{H}), 1707,1661(\mathrm{C}=\mathrm{O}) \mathrm{cm}^{-1}$; ${ }^{1} \mathrm{H}$ NMR (DMSO$\left.d_{6}, 500 \mathrm{MHz}\right): \delta=0.88\left(3 \mathrm{H}, \mathrm{t}, J=7.3 \mathrm{~Hz}, 8-\mathrm{CH}_{2} \mathrm{CH}_{2} \mathrm{CH}_{3}-\right.$ sp.), 1.05-1.34 (7H, m, CH/CH - -sp., 8- $\mathrm{CH}_{2} \mathrm{CH}_{2} \mathrm{CH}_{3}$-sp.), 1.60-1.82 (6H, m, CH/CH ${ }_{2}$-sp.), $3.58\left(2 \mathrm{H}, \mathrm{s}, \mathrm{S}-\mathrm{CH}_{2}-\right.$ sp.), 7.29 (1H, dd, $J=8.8,2.0 \mathrm{~Hz}, \mathrm{H6}-\mathrm{ind}$.), $7.35(1 \mathrm{H}, \mathrm{tt}$, $J=7.3,1.5 \mathrm{~Hz}, 3-\mathrm{C}_{6} \mathrm{H}_{5}(\mathrm{H} 4)$-ind.), $7.45(2 \mathrm{H}, \mathrm{t}, J=7.8 \mathrm{~Hz}$, $3-\mathrm{C}_{6} \mathrm{H}_{5}(\mathrm{H} 3, \mathrm{H} 5)$-ind.), $7.51(1 \mathrm{H}, \mathrm{d}, J=2.0 \mathrm{~Hz}, \mathrm{H} 4$-ind.), 7.53-7.56 (3H, m, H7, 3- $\mathrm{C}_{6} \mathrm{H}_{5}(\mathrm{H} 2, \mathrm{H} 6)$-ind.), $10.10(1 \mathrm{H}, \mathrm{s}$, $\mathrm{CONH}), 12.13(1 \mathrm{H}, \mathrm{s}, \mathrm{NH}) \mathrm{ppm} ;{ }^{13} \mathrm{C}$ NMR (APT, DMSO$\left.d_{6}, 125 \mathrm{MHz}\right): \delta=14.58\left(8-\mathrm{CH}_{2} \mathrm{CH}_{2} \mathrm{CH}_{3}\right.$-sp.), 19.99, 28.30, 28.36, $29.77\left(\mathrm{CH}_{2}\right.$-sp., 8- $\mathrm{CH}_{2} \mathrm{CH}_{2} \mathrm{CH}_{3}$-sp.), 35.25 (C8-sp.), 36.85, $38.76\left(\mathrm{CH}_{2}\right.$-sp., 8- $\mathrm{CH}_{2} \mathrm{CH}_{2} \mathrm{CH}_{3}$-sp.), 72.74 (C5-sp.), 
114.55, 114.61 (CH-ar.), 118.47 (C-ar.), 119.30, 119.38 (CH-ar.), 124.54 (CH-ar.), 125.47 (C-ar.), 127.30 (CH-ar.), 127.67 (C-ar.), 128.28 (C-ar.), 128.84 (CH-ar.), 130.51 (CHar.), 133.42 (C-ar.), 134.61 (C-ar.), 162.12 (CO-NH), 167.77 (CO-sp.) ppm.

5-Chloro- $N$-(7,7,9-trimethyl-3-oxo-1-thia-4-azaspiro[4.5]decan-4-yl)-3-phenyl- $1 \mathrm{H}$-indole-2-carboxamide $(\mathbf{4 g}$, $\mathrm{C}_{26} \mathrm{H}_{28} \mathrm{CIN}_{3} \mathrm{O}_{2} \mathrm{~S}$ ) White crystals; yield: $85.1 \%$; m.p.: 264$266.5^{\circ} \mathrm{C} ; R_{f}=0.56\left(\mathrm{EtOAc} \mathrm{CHCl}_{3} 1: 1\right)$; IR $(\mathrm{KBr}): \bar{v}=3277$, $3219(\mathrm{~N}-\mathrm{H}), 1705,1695,1665(\mathrm{C}=\mathrm{O}) \mathrm{cm}^{-1} ;{ }^{1} \mathrm{H}$ NMR (DMSO- $\left.d_{6}, 500 \mathrm{MHz}\right): \delta=0.76(1 \mathrm{H}, \mathrm{t}, J=12.7 \mathrm{~Hz}, \mathrm{CH} /$ $\mathrm{CH}_{2}$-sp.), 0.90 (6H, s*, 7-( $\left.\mathrm{CH}_{3}\right)_{2}$-sp.), 1.04 (3H, s*, 9- $\mathrm{CH}_{3}-$ sp.), $1.20-1.34\left(2 \mathrm{H}, \mathrm{m}, \mathrm{CH} / \mathrm{CH}_{2}\right.$-sp.), $1.58-1.84$ (4H, m, CH/ $\mathrm{CH}_{2}$-sp.), $3.50\left(1 \mathrm{H}, \mathrm{d}, J=16.1 \mathrm{~Hz}, \mathrm{~S}-\mathrm{CH}_{2}\right.$-sp.), $3.61(1 \mathrm{H}$, d, $J=16.1 \mathrm{~Hz}, \mathrm{~S}-\mathrm{CH}_{2}$-sp.), $7.29(1 \mathrm{H}, \mathrm{dd}, J=8.6,2.0 \mathrm{~Hz}$, H6-ind.), 7.37 (1H, t*, $J=7.3 \mathrm{~Hz}, 3-\mathrm{C}_{6} \mathrm{H}_{5}(\mathrm{H} 4)$-ind.), 7.44$7.48\left(3 \mathrm{H}, \mathrm{m}, \mathrm{H} 4,3-\mathrm{C}_{6} \mathrm{H}_{5}(\mathrm{H} 3, \mathrm{H} 5)-\right.$ ind. $), 7.51-7.54(3 \mathrm{H}$, m, H7, 3- $\mathrm{C}_{6} \mathrm{H}_{5}(\mathrm{H} 2, \mathrm{H} 6)$-ind.), $9.97(1 \mathrm{H}, \mathrm{s}, \mathrm{CONH}), 12.13$ $(1 \mathrm{H}, \mathrm{s}, \mathrm{NH}) \mathrm{ppm} ;{ }^{13} \mathrm{C}$ NMR (APT, DMSO- $d_{6}, 125 \mathrm{MHz}$ ): $\delta=22.24,25.97,27.64\left(7-\left(\mathrm{CH}_{3}\right)_{2}, 9-\mathrm{CH}_{3}\right.$-sp. $), 28.50,32.24$ ( $\mathrm{CH}_{2}$-sp.), 34.09 (C9-sp.), 45.23, 46.80, $47.31\left(\mathrm{CH}_{2}\right.$-sp., C7-sp.), 71.87 (C5-sp.), 114.56, 114.64 (CH-ar.), 118.50 (C-ar.), 119.28, 119.38 (CH-ar.), 124.58 (CH-ar.), 125.45 (C-ar.), 127.44 (CH-ar.), 127.96 (C-ar.), 128.18 (C-ar.), 128.99 (CH-ar.), 130.54 (CH-ar.), 133.47 (C-ar.), 134.62 (C-ar.), 162.40 (CO-NH), 167.64 (CO-sp.) ppm.

5-Chloro-3-phenyl- $\mathrm{N}$-(8-phenyl-3-oxo-1-thia-4-azaspiro[4.5]decan-4-yl)-1 H-indole-2-carboxamide (4h, $\mathrm{C}_{29} \mathrm{H}_{26} \mathrm{CIN}_{3} \mathrm{O}_{2} \mathrm{~S}$ ) Beige powder; yield: 72.8\%; m.p.: 285$287{ }^{\circ} \mathrm{C} ; R_{f}=0.58\left(\mathrm{EtOAc} / \mathrm{CHCl}_{3} 1: 1\right)$; IR (KBr): $\bar{v}=3277$, $3237(\mathrm{~N}-\mathrm{H}), 1707,1663(\mathrm{C}=\mathrm{O}) \mathrm{cm}^{-1}$; ${ }^{1} \mathrm{H}$ NMR (DMSO$\left.d_{6}, 500 \mathrm{MHz}\right): \delta=1.60-1.91\left(8 \mathrm{H}, \mathrm{m}, \mathrm{CH}_{2}\right.$-sp.), 2.47-2.49 (1H, m, CH-sp.), 3.64 (2H, s, S- $\mathrm{CH}_{2}$-sp.), $7.21\left(1 \mathrm{H}, \mathrm{tt}^{*}\right.$, $J=7.3,1.5 \mathrm{~Hz}, 8-\mathrm{C}_{6} \mathrm{H}_{5}(\mathrm{H} 4)$-sp.), $7.25\left(2 \mathrm{H}, \mathrm{d}^{*}, J=7.3 \mathrm{~Hz}\right.$, 8- $\mathrm{C}_{6} \mathrm{H}_{5}$-sp.), 7.29-7.34 (3H, m, H6-ind., 8- $\mathrm{C}_{6} \mathrm{H}_{5}$-sp.), 7.41 $\left(1 \mathrm{H}, \mathrm{tt}^{*}, J=7.8,1.5 \mathrm{~Hz}, 3-\mathrm{C}_{6} \mathrm{H}_{5}(\mathrm{H} 4)\right.$-ind.), $7.50-7.61(6 \mathrm{H}$, $\mathrm{m}, \mathrm{H} 4, \mathrm{H} 7,3-\mathrm{C}_{6} \mathrm{H}_{5}(\mathrm{H} 2, \mathrm{H} 3, \mathrm{H} 5, \mathrm{H} 6)$-ind.), $10.18(1 \mathrm{H}, \mathrm{s}$, $\mathrm{CONH}), 12.17(1 \mathrm{H}, \mathrm{s}, \mathrm{NH}) \mathrm{ppm} ;{ }^{13} \mathrm{C}$ NMR (APT, DMSO$\left.d_{6}, 125 \mathrm{MHz}\right): \delta=28.38,30.92,37.12\left(\mathrm{CH}_{2}\right.$-sp. $), 41.95$ (C8sp.), 72.10 (C5-sp.), 114.57, 114.64 (CH-ar.), 118.60 (C-ar.), 119.34, 119.42 (CH-ar.), 124.62 (CH-ar.), 125.51 (C-ar.), 126.98 (8-ar.(CH)-sp.), 127.09 (CH-ar.), 127.73 (8-ar.(CH)sp.), 127.88 (C-ar.), 128.28 (C-ar.), 128.95 (CH-ar.), 130.57 (CH-ar.), 133.45 (C-ar.), 134.63 (C-ar.), 146.26 (8-ar.(C)sp.), 162.18 (CO-NH), 167.84 (CO-sp.) ppm.

$N$-(8-tert-Butyl-3-oxo-1-thia-4-azaspiro [4.5]decan-4-yl)-5-chloro-3-phenyl-1 $\mathrm{H}$-indole-2-carboxamide $\left(4 \mathrm{i}, \mathrm{C}_{27} \mathrm{H}_{30} \mathrm{CIN}_{3} \mathrm{O}_{2} \mathrm{~S}\right)$ White crystals; yield: 79.3\%; m.p.: 230$232{ }^{\circ} \mathrm{C} ; R_{f}=0.57\left(\mathrm{EtOAc} / \mathrm{CHCl}_{3} 1: 1\right)$; IR (KBr): $\bar{v}=3285$ $(\mathrm{N}-\mathrm{H}), 1702,1662(\mathrm{C}=\mathrm{O}) \mathrm{cm}^{-1} ;{ }^{1} \mathrm{H}$ NMR (DMSO- $d_{6}$,
$500 \mathrm{MHz}): \delta=0.84$ (9H, s, 8-C( $\left(\mathrm{CH}_{3}\right)_{3}$-sp. $), 0.90-0.96(1 \mathrm{H}$, $\mathrm{m}, \mathrm{CH} / \mathrm{CH}_{2}$-sp.), 1.12-1.22 (2H, m, CH/CH ${ }_{2}$-sp.), 1.64-1.72 (6H, m, CH/CH $\mathrm{CH}_{2}$-sp.), $3.58\left(2 \mathrm{H}, \mathrm{s}, \mathrm{S}-\mathrm{CH}_{2}\right.$-sp.), $7.30(1 \mathrm{H}$, $\mathrm{dd}, J=8.8,2.0 \mathrm{~Hz}, \mathrm{H} 6$-ind. $), 7.36(1 \mathrm{H}, \mathrm{tt}, J=7.3,1.5 \mathrm{~Hz}$, $3-\mathrm{C}_{6} \mathrm{H}_{5}(\mathrm{H} 4)$-ind.), $7.45\left(2 \mathrm{H}, \mathrm{t}, J=7.3 \mathrm{~Hz}, 3-\mathrm{C}_{6} \mathrm{H}_{5}(\mathrm{H} 3, \mathrm{H} 5)\right.$ ind.), $7.50(1 \mathrm{H}, \mathrm{d}, J=2.0 \mathrm{~Hz}, \mathrm{H} 4-\mathrm{ind}$.), 7.52-7.56 (3H, m, H7, 3- $\mathrm{C}_{6} \mathrm{H}_{5}(\mathrm{H} 2, \mathrm{H} 6)$-ind.), $10.13(1 \mathrm{H}, \mathrm{s}, \mathrm{CONH}), 12.14$ $(1 \mathrm{H}, \mathrm{s}, \mathrm{NH}) \mathrm{ppm} ;{ }^{13} \mathrm{C}$ NMR (APT, DMSO- $d_{6}, 125 \mathrm{MHz}$ ): $\delta=24.14\left(\mathrm{CH}_{2}\right.$-sp.), $27.80\left(8-\mathrm{C}\left(\mathrm{CH}_{3}\right)_{3}\right.$-sp. $), 28.28\left(\mathrm{CH}_{2}\right.$-sp.), 32.39 (8- $\mathrm{C}\left(\mathrm{CH}_{3}\right)_{3}$-sp.), $37.11\left(\mathrm{CH}_{2}\right.$-sp.), 45.96 (C8-sp.), 72.57 (C5-sp.), 114.60 (CH-ar.), 118.42 (C-ar.), 119.27, 119.35 (CH-ar.), 124.64 (CH-ar.), 125.45 (C-ar.), 127.40 (CH-ar.), 127.84 (C-ar.), 128.38 (C-ar.), 128.90 (CH-ar.), 130.51 (CH-ar.), 133.44 (C-ar.), 134.58 (C-ar.), 162.13 (CO$\mathrm{NH}), 167.79$ (CO-sp.) ppm; MS (ESI-): $\mathrm{m} / z(\%)=494.5$ ([M$\left.\mathrm{H}]^{-}, 100\right), 496.3\left([(\mathrm{M}-\mathrm{H})+2]^{-}, 37.6\right)$.

5-Chloro- $\mathrm{N}$-(2-methyl-3-oxo-1-thia-4-azaspiro[4.4]nonan-4-yl)-3-phenyl-1H-indole-2-carboxamide (5a, $\mathrm{C}_{23} \mathrm{H}_{22} \mathrm{CIN}_{3} \mathrm{O}_{2} \mathrm{~S}$ ) Beige powder; yield: $85.4 \%$; m.p.: 270 $272{ }^{\circ} \mathrm{C} ; R_{f}=0.60\left(\mathrm{EtOAc} / \mathrm{CHCl}_{3} 1: 1\right) ; \mathrm{IR}(\mathrm{KBr}): \bar{v}=3283$ $(\mathrm{N}-\mathrm{H}), 1709,1651(\mathrm{C}=\mathrm{O}) \mathrm{cm}^{-1} ;{ }^{1} \mathrm{H}$ NMR (DMSO- $d_{6}$, $500 \mathrm{MHz}): \delta=1.42\left(3 \mathrm{H}, \mathrm{d}, J=6.8 \mathrm{~Hz}, 2-\mathrm{CH}_{3}\right.$-sp.), 1.59 1.77 (6H, m, $\mathrm{CH}_{2}$-sp.), 1.91-2.08 (2H, m, $\mathrm{CH}_{2}$-sp.), 3.93 $(1 \mathrm{H}, \mathrm{q}, J=6.8 \mathrm{~Hz}, \mathrm{~S}-\mathrm{CH}-\mathrm{sp}),. 7.29(1 \mathrm{H}, \mathrm{dd}, J=8.8,2.0 \mathrm{~Hz}$, H6-ind.), $7.36\left(1 \mathrm{H}, \mathrm{tt}, J=7.8,1.5 \mathrm{~Hz}, 3-\mathrm{C}_{6} \mathrm{H}_{5}(\mathrm{H} 4)\right.$-ind.), $7.44\left(2 \mathrm{H}, \mathrm{t}, J=7.8 \mathrm{~Hz}, 3-\mathrm{C}_{6} \mathrm{H}_{5}(\mathrm{H} 3, \mathrm{H} 5)\right.$-ind.), 7.50-7.55 (4H, m, H4, H7, 3- $\mathrm{C}_{6} \mathrm{H}_{5}(\mathrm{H} 2, \mathrm{H} 6)$-ind.), $10.14(1 \mathrm{H}, \mathrm{s}$, $\mathrm{CONH}), 12.13(1 \mathrm{H}, \mathrm{s}, \mathrm{NH}) \mathrm{ppm} ;{ }^{13} \mathrm{C}$ NMR (APT, DMSO$\left.d_{6}, 125 \mathrm{MHz}\right): \delta=19.81\left(2-\mathrm{CH}_{3}\right.$-sp.), $22.47\left(\mathrm{CH}_{2}\right.$-sp. $)$, 37.93, 38.06 (C2-sp.), 38.25, $38.37\left(\mathrm{CH}_{2}\right.$-sp.), 74.96 (C5sp.), 114.60, 114.66 (CH-ar.), 118.64 (C-ar.), 119.33, 119.41 (CH-ar.), 124.63, 124.74 (CH-ar.), 125.49 (C-ar.), 127.48 (CH-ar.), 127.84 (C-ar.), 128.02 (C-ar.), 128.81 (CH-ar.), 130.48 (CH-ar.), 133.27 (C-ar.), 134.65 (C-ar.), 162.05 (CONH), 170.57 (CO-sp.) ppm.

5-Chloro- $\mathrm{N}$-(2-methyl-3-oxo-1-thia-4-azaspiro[4.5]decan-4-yl)-3-phenyl-1 $\mathrm{H}$-indole-2-carboxamide (5b, $\left.\mathrm{C}_{24} \mathrm{H}_{24} \mathrm{ClN}_{3} \mathrm{O}_{2} \mathrm{~S}\right)$ White crystals; yield: $90.7 \%$; m.p.: $245-$ $248{ }^{\circ} \mathrm{C} ; R_{f}=0.62\left(\mathrm{EtOAc} / \mathrm{CHCl}_{3} 1: 1\right)$; IR (KBr): $\bar{v}=3285$ $(\mathrm{N}-\mathrm{H}), 1711,1651(\mathrm{C}=\mathrm{O}) \mathrm{cm}^{-1} ;{ }^{1} \mathrm{H}$ NMR (DMSO- $d_{6}$, $500 \mathrm{MHz}): \delta=1.02-1.11\left(2 \mathrm{H}, \mathrm{m}, \mathrm{CH}_{2}\right.$-sp.), $1.31-1.38(2 \mathrm{H}$, $\mathrm{m}, \mathrm{CH}_{2}$-sp.), 1.41 (3H, d, $J=6.8 \mathrm{~Hz}, 2-\mathrm{CH}_{3}$-sp.), $1.56(1 \mathrm{H}$, d, $J=12.7 \mathrm{~Hz}, \mathrm{CH}_{2}$-sp.), 1.62-1.75 (5H, m, $\mathrm{CH}_{2}$-sp.), 3.93 $(1 \mathrm{H}, \mathrm{q}, J=6.4 \mathrm{~Hz}, \mathrm{~S}-\mathrm{CH}-\mathrm{sp}),. 7.30(1 \mathrm{H}, \mathrm{dd}, J=8.8,2.0 \mathrm{~Hz}$, H6-ind.), 7.37 (1H, t, $J=7.3 \mathrm{~Hz}, 3-\mathrm{C}_{6} \mathrm{H}_{5}(\mathrm{H} 4)$-ind.), 7.47 $\left(2 \mathrm{H}, \mathrm{t}, J=7.3 \mathrm{~Hz}, 3-\mathrm{C}_{6} \mathrm{H}_{5}(\mathrm{H} 3, \mathrm{H} 5)\right.$-ind.) $7.50-7.55(4 \mathrm{H}, \mathrm{m}$, $\mathrm{H} 4, \mathrm{H} 7,3-\mathrm{C}_{6} \mathrm{H}_{5}(\mathrm{H} 2, \mathrm{H} 6)$-ind.), 10.12 (1H, s, $\left.\mathrm{CONH}\right), 12.14$ $(1 \mathrm{H}, \mathrm{s}, \mathrm{NH}) \mathrm{ppm} ;{ }^{13} \mathrm{C}$ NMR (APT, DMSO- $d_{6}, 125 \mathrm{MHz}$ ): $\delta=20.01\left(2-\mathrm{CH}_{3}\right.$-sp.), 23.59, $24.31\left(\mathrm{CH}_{2}\right.$-sp.), $37.12(\mathrm{C} 2-$ sp.), $38.02\left(\mathrm{CH}_{2}\right.$-sp.), 71.29 (C5-sp.), 114.56, 114.63 (CH-ar.), 118.50 (C-ar.), 119.31, 119.39 (CH-ar.), 124.63 
(CH-ar.), 125.46 (C-ar.), 127.42 (CH-ar.), 127.84 (C-ar.), 128.23 (C-ar.), 128.90 (CH-ar.), 130.54 (CH-ar.), 133.37 (C-ar.), 134.62 (C-ar.), 162.11 (CO-NH), 170.36 (CO-sp.) ppm.

5-Chloro- $N$-(2,7-dimethyl-3-oxo-1-thia-4-azaspiro[4.5] decan-4-yl)-3-phenyl- $1 \mathrm{H}$-indole-2-carboxamide (5c, $\mathrm{C}_{25} \mathrm{H}_{26} \mathrm{CIN}_{3} \mathrm{O}_{2} \mathrm{~S}$ ) White crystals; yield: $86.0 \%$; m.p.: 262$264{ }^{\circ} \mathrm{C} ; R_{f}=0.65\left(\mathrm{EtOAc} / \mathrm{CHCl}_{3} 1: 1\right) ; \mathrm{IR}(\mathrm{KBr}): \bar{v}=3235$ $(\mathrm{N}-\mathrm{H}), 1694,1661(\mathrm{C}=\mathrm{O}) \mathrm{cm}^{-1} ;{ }^{1} \mathrm{H}$ NMR (DMSO- $d_{6}$, $500 \mathrm{MHz}): \delta=0.75-0.80\left(1 \mathrm{H}, \mathrm{m}, \mathrm{CH} / \mathrm{CH}_{2}\right.$-sp. $), 0.87$ $\left(3 \mathrm{H}, \mathrm{d}, J=6.0 \mathrm{~Hz}, 7-\mathrm{CH}_{3}\right.$-sp.), $1.23-1.68(8 \mathrm{H}, \mathrm{m}, \mathrm{CH} /$ $\mathrm{CH}_{2}$-sp.), $1.41\left(3 \mathrm{H}, \mathrm{d}, J=6.3 \mathrm{~Hz}, 2-\mathrm{CH}_{3}\right.$-sp. $), 3.88(1 \mathrm{H}$, s*, S-CH-sp.), 7.29 (1H, dd, $J=8.6,2.0 \mathrm{~Hz}, \mathrm{H} 6$-ind.), $7.37\left(1 \mathrm{H}, \mathrm{tt}, J=7.3,1.5 \mathrm{~Hz}, 3-\mathrm{C}_{6} \mathrm{H}_{5}(\mathrm{H} 4)\right.$-ind.), 7.45-7.49 $\left(3 \mathrm{H}, \mathrm{m}, \mathrm{H} 4,3-\mathrm{C}_{6} \mathrm{H}_{5}(\mathrm{H} 3, \mathrm{H} 5)-i n d.\right), 7.52-7.54(3 \mathrm{H}, \mathrm{m}, \mathrm{H} 7$, 3- $\mathrm{C}_{6} \mathrm{H}_{5}(\mathrm{H} 2, \mathrm{H} 6)$-ind.), $10.07(1 \mathrm{H}, \mathrm{s}, \mathrm{CONH}), 12.12(1 \mathrm{H}, \mathrm{s}$, $\mathrm{NH}$ ) ppm; ${ }^{13} \mathrm{C}$ NMR (APT, DMSO- $d_{6}, 125 \mathrm{MHz}$ ): $\delta=20.01$,

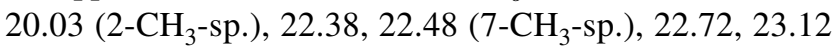
( $\mathrm{CH}_{2}$-sp.), 29.86, 30.25 (C7-sp.), 32.98, $36.66\left(\mathrm{CH}_{2}\right.$-sp.), 37.27, 37.38 (C2-sp.), 37.71, 45.41, $46.10\left(\mathrm{CH}_{2}\right.$-sp.), 71.22, 71.26 (C5-sp.), 114.55, 114.63 (CH-ar.), 118.52 (C-ar.), 119.29, 119.39 (CH-ar.), 124.56, 124.65 (CH-ar.), 125.45 (C-ar.), 127.42 (CH-ar.), 127.88 (C-ar.), 128.25 (C-ar.), 128.90 (CH-ar.), 130.55 (CH-ar.), 133.43 (C-ar.), 134.62 (C-ar.), 162.14 (CO-NH), 170.30 (CO-sp.) ppm; MS (ESI-): $m / z(\%)=466.7\left([\mathrm{M}-\mathrm{H}]^{-}, 100\right), 468.5\left([(\mathrm{M}-\mathrm{H})+2]^{-}, 33.4\right)$.

5-Chloro- $\mathrm{N}$-(2,8-dimethyl-3-oxo-1-thia-4-azaspiro[4.5]decan-4-yl)-3-phenyl- $1 \mathrm{H}$-indole-2-carboxamide (5d, $\left.\mathrm{C}_{25} \mathrm{H}_{26} \mathrm{ClN}_{3} \mathrm{O}_{2} \mathrm{~S}\right)$ White crystals; yield: $95.1 \%$; m.p.: 223$225.5^{\circ} \mathrm{C} ; R_{f}=0.65\left(\mathrm{EtOAc} / \mathrm{CHCl}_{3} 1: 1\right)$; IR (KBr): $\bar{v}=3337$, $3262(\mathrm{~N}-\mathrm{H}), 1694,1670(\mathrm{C}=\mathrm{O}) \mathrm{cm}^{-1} ;{ }^{1} \mathrm{H}$ NMR (DMSO- $d_{6}$, $500 \mathrm{MHz}): \delta=0.89,0.96$ (3H, 2d, $J=6.4 \mathrm{~Hz}, 8-\mathrm{CH}_{3}$-sp.), $1.03-1.36\left(3 \mathrm{H}, \mathrm{m}, \mathrm{CH} / \mathrm{CH}_{2}\right.$-sp.), $1.41(3 \mathrm{H}, \mathrm{d}, J=6.8 \mathrm{~Hz}$, 2- $\mathrm{CH}_{3}$-sp.), 1.50-1.74 (6H, m, CH/CH ${ }_{2}$-sp.), $3.87\left(1 \mathrm{H}, \mathrm{q}^{*}\right.$, $J=6.8 \mathrm{~Hz}, \mathrm{~S}-\mathrm{CH}-\mathrm{sp}$.), 7.30, $7.31(1 \mathrm{H}, 2 \mathrm{dd}, J=8.8,2.0 \mathrm{~Hz}$, H6-ind.), $7.37\left(1 \mathrm{H}, \mathrm{tt}, J=7.3,1.5 \mathrm{~Hz}, 3-\mathrm{C}_{6} \mathrm{H}_{5}(\mathrm{H} 4)\right.$-ind.), $7.46\left(2 \mathrm{H}, \mathrm{t}, J=7.3 \mathrm{~Hz}, 3-\mathrm{C}_{6} \mathrm{H}_{5}(\mathrm{H} 3, \mathrm{H} 5)\right.$-ind. $), 7.50(1 \mathrm{H}, \mathrm{d}$, $J=2.0 \mathrm{~Hz}, \mathrm{H} 4$-ind.), 7.52-7.58 (3H, m, H7, 3- $\mathrm{C}_{6} \mathrm{H}_{5}(\mathrm{H} 2, \mathrm{H} 6)$ ind.), $10.10(1 \mathrm{H}, \mathrm{s}, \mathrm{CONH}), 12.13(1 \mathrm{H}, \mathrm{s}, \mathrm{NH}) \mathrm{ppm} ;{ }^{13} \mathrm{C}$ NMR (proton decoupled and DEPT, DMSO- $d_{6}, 75 \mathrm{MHz}$ ):

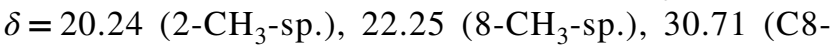
sp.), 31.60, 32.02, 36.91 ( $\mathrm{CH}_{2}$-sp.), 37.10 (C2-sp.), 37.78 ( $\mathrm{CH}_{2}$-sp.), 71.05 (C5-sp.), 114.50 (CH-ar.), 118.43 (C-ar.), 119.26 (CH-ar.), 124.52 (CH-ar.), 125.38 (C-ar.), 127.27 (CH-ar.), 127.75 (C-ar.), 128.08 (C-ar.), 128.76 (CH-ar.), 130.41 (CH-ar.), 133.27 (C-ar.), 134.53 (C-ar.), 161.98 (CO$\mathrm{NH}), 170.33$ (CO-sp.) ppm.

5-Chloro- $N$-(8-ethyl-2-methyl-3-oxo-1-thia-4-azaspiro[4.5]decan-4-yl)-3-phenyl- $1 \mathrm{H}$-indole-2-carboxamide (5e, $\mathrm{C}_{26} \mathrm{H}_{28} \mathrm{CIN}_{3} \mathrm{O}_{2} \mathrm{~S}$ ) White needles; yield: $87.2 \%$; m.p.:
198-200 ${ }^{\circ} \mathrm{C} ; R_{f}=0.64\left(\mathrm{EtOAc} / \mathrm{CHCl}_{3} 1: 1\right)$; IR (KBr): $\bar{v}=3383(\mathrm{~N}-\mathrm{H}), 1711,1653(\mathrm{C}=\mathrm{O}) \mathrm{cm}^{-1}$; ${ }^{1} \mathrm{H}$ NMR (DMSO$d_{6}, 500 \mathrm{MHz}$ ): $\delta=0.89$ (3H, t, $J=7.3 \mathrm{~Hz}, 8-\mathrm{CH}_{2} \mathrm{CH}_{3}$-sp.), 1.02-1.24 (5H, m, CH/CH ${ }_{2}$-sp., 8- $\mathrm{CH}_{2} \mathrm{CH}_{3}$-sp.), $1.41(3 \mathrm{H}, \mathrm{d}$, $J=6.8 \mathrm{~Hz}, 2-\mathrm{CH}_{3}$-sp.), $1.62-1.88\left(6 \mathrm{H}, \mathrm{m}, \mathrm{CH} / \mathrm{CH}_{2}\right.$-sp.), 3.87 $\left(1 \mathrm{H}, \mathrm{q}^{*}, J=6.3 \mathrm{~Hz}, \mathrm{~S}-\mathrm{CH}-\mathrm{sp}.\right), 7.30(1 \mathrm{H}, \mathrm{dd}, J=8.8,2.0 \mathrm{~Hz}$, H6-ind.), 7.37 (1H, tt, $J=7.8,1.5 \mathrm{~Hz}, 3-\mathrm{C}_{6} \mathrm{H}_{5}(\mathrm{H} 4)$-ind.), $7.46\left(2 \mathrm{H}, \mathrm{t}, J=7.8 \mathrm{~Hz}, 3-\mathrm{C}_{6} \mathrm{H}_{5}(\mathrm{H} 3, \mathrm{H} 5)-\right.$ ind. $), 7.51(1 \mathrm{H}, \mathrm{d}$, $J=2.0 \mathrm{~Hz}, \mathrm{H} 4-$ ind.), 7.52-7.56 (3H, m, H7, 3- $\mathrm{C}_{6} \mathrm{H}_{5}(\mathrm{H} 2, \mathrm{H} 6)-$ ind.), $10.13(1 \mathrm{H}, \mathrm{s}, \mathrm{CONH}), 12.13(1 \mathrm{H}, \mathrm{s}, \mathrm{NH}) \mathrm{ppm} ;{ }^{13} \mathrm{C}$ NMR (APT, DMSO- $\left.d_{6}, 125 \mathrm{MHz}\right): \delta=11.92\left(8-\mathrm{CH}_{2} \mathrm{CH}_{3}-\right.$ sp.), 20.35 (2- $\mathrm{CH}_{3}$-sp.), 29.23, 29.67, $36.98\left(\mathrm{CH}_{2}\right.$-sp., 8- $\mathrm{CH}_{2} \mathrm{CH}_{3}$-sp.), 37.14, 37.27 (C2-sp.), 37.42 (C8-sp.), 37.85 ( $\mathrm{CH}_{2}$-sp., 8 - $\mathrm{CH}_{2} \mathrm{CH}_{3}$-sp.), 71.42, 71.71 (C5-sp.), 114.55, 114.61 (CH-ar.), 118.50 (C-ar.), 119.32, 119.39 (CH-ar.), 124.56, 124.64 (CH-ar.), 125.46 (C-ar.), 127.39 (CH-ar.), 127.85 (C-ar.), 128.24 (C-ar.), 128.88 (CH-ar.), 130.51 (CHar.), 133.39 (C-ar.), 134.60 (C-ar.), 162.07 (CO-NH), 170.41 (CO-sp.) ppm.

5-Chloro-N-(2-methyl-8-propyl-3-oxo-1-thia-4-azaspiro[4.5]decan-4-yl)-3-phenyl-1 $\mathrm{H}$-indole-2-carboxamide (5f, $\left.\mathrm{C}_{27} \mathrm{H}_{30} \mathrm{CIN}_{3} \mathrm{O}_{2} \mathrm{~S}\right)$ Beige crystals; yield: $89.1 \%$; m.p.: 194-197 ${ }^{\circ} \mathrm{C} ; R_{f}=0.67\left(\mathrm{EtOAc} / \mathrm{CHCl}_{3} 1: 1\right)$; IR (KBr): $\bar{v}=3287,3184(\mathrm{~N}-\mathrm{H}), 1713,1692,1653(\mathrm{C}=\mathrm{O}) \mathrm{cm}^{-1} ;{ }^{1} \mathrm{H}$ NMR (DMSO- $\left.d_{6}, 500 \mathrm{MHz}\right): \delta=0.88(3 \mathrm{H}, \mathrm{t}, J=7.3 \mathrm{~Hz}$, 8- $\mathrm{CH}_{2} \mathrm{CH}_{2} \mathrm{CH}_{3}$-sp.), 1.00-1.34 (7H, m, CH/CH - -sp., 8- $\mathrm{CH}_{2} \mathrm{CH}_{2} \mathrm{CH}_{3}$-sp.), $1.42\left(3 \mathrm{H}, \mathrm{d}, J=6.8 \mathrm{~Hz}, 2-\mathrm{CH}_{3}\right.$-sp.), 1.60-1.84 (6H, m, CH/CH ${ }_{2}$-sp.), $3.89\left(1 \mathrm{H}, \mathrm{d}^{*}, J=5.8 \mathrm{~Hz}\right.$, S-CH-sp.), 7.30 (1H, dd, $J=8.8,2.0 \mathrm{~Hz}, \mathrm{H6}$-ind.), 7.36 $\left(1 \mathrm{H}, \mathrm{tt}, J=7.3,1.5 \mathrm{~Hz}, 3-\mathrm{C}_{6} \mathrm{H}_{5}(\mathrm{H} 4)-\right.$ ind. $), 7.45(2 \mathrm{H}, \mathrm{t}$, $J=7.3 \mathrm{~Hz}, 3-\mathrm{C}_{6} \mathrm{H}_{5}(\mathrm{H} 3, \mathrm{H} 5)$-ind. $), 7.50(1 \mathrm{H}, \mathrm{d}, J=2.0 \mathrm{~Hz}$, H4-ind.), 7.52-7.56 (3H, m, H7, 3- $\mathrm{C}_{6} \mathrm{H}_{5}(\mathrm{H} 2, \mathrm{H} 6)$-ind.), 10.13 $(1 \mathrm{H}, \mathrm{s}, \mathrm{CONH}), 12.13(1 \mathrm{H}, \mathrm{s}, \mathrm{NH}) \mathrm{ppm} ;{ }^{13} \mathrm{C} \mathrm{NMR}$ (APT, DMSO- $\left.d_{6}, 125 \mathrm{MHz}\right): \delta=14.57\left(8-\mathrm{CH}_{2} \mathrm{CH}_{2} \mathrm{CH}_{3}\right.$-sp.), 20.00 ( $\mathrm{CH}_{2}$-sp., 8- $\mathrm{CH}_{2} \mathrm{CH}_{2} \mathrm{CH}_{3}$-sp.), 20.31 (2- $\mathrm{CH}_{3}$-sp.), 29.62, 30.05 ( $\mathrm{CH}_{2}$-sp., 8- $\mathrm{CH}_{2} \mathrm{CH}_{2} \mathrm{CH}_{3}$-sp.), 35.26 (C8-sp.), 37.00 ( $\mathrm{CH}_{2}$-sp., 8- $\mathrm{CH}_{2} \mathrm{CH}_{2} \mathrm{CH}_{3}$-sp.), 37.16, 37.29 (C2-sp.), 37.86, $38.79\left(\mathrm{CH}_{2}\right.$-sp., 8- $\mathrm{CH}_{2} \mathrm{CH}_{2} \mathrm{CH}_{3}$-sp.), 71.39, 71.75 (C5-sp.), 114.53, 114.60 (CH-ar.), 118.50 (C-ar.), 119.32, 119.39 (CH-ar.), 124.55, 124.64 (CH-ar.), 125.47, 125.52 (C-ar.), 127.36 (CH-ar.), 127.66 (C-ar.), 128.27 (C-ar.), 128.84 (CH-ar.), 130.55 (CH-ar.), 133.32, 133.43 (C-ar.), 134.61, 134.66 (C-ar.), 162.08 (CO-NH), 170.37, 170.41 (CO-sp.) ppm; MS (ESI-): $\mathrm{m} / \mathrm{z}(\%)=494.7\left([\mathrm{M}-\mathrm{H}]^{-}, 100\right), 496.4$ $\left([(\mathrm{M}-\mathrm{H})+2]^{-}, 34.2\right)$.

5-Chloro- $\mathrm{N}$-(2,7,7,9-tetramethyl-3-oxo-1-thia-4-azaspiro[4.5]decan-4-yl)-3-phenyl- $1 \mathrm{H}$-indole-2-carboxamide $\mathbf{~} 5 \mathrm{~g}$, $\mathrm{C}_{27} \mathrm{H}_{30} \mathrm{CIN}_{3} \mathrm{O}_{2} \mathrm{~S}$ ) White crystals; yield: 75.5\%; m.p.: 266$268{ }^{\circ} \mathrm{C} ; R_{f}=0.68\left(\mathrm{EtOAc} / \mathrm{CHCl}_{3} 1: 1\right) ; \mathrm{IR}(\mathrm{KBr}): \bar{v}=3287$ $(\mathrm{N}-\mathrm{H}), 1705,1655(\mathrm{C}=\mathrm{O}) \mathrm{cm}^{-1} ;{ }^{1} \mathrm{H}$ NMR (DMSO- $d_{6}$, $500 \mathrm{MHz}): \delta=0.76\left(1 \mathrm{H}, \mathrm{t}, J=12.7 \mathrm{~Hz}, \mathrm{CH} / \mathrm{CH}_{2}\right.$-sp. $), 0.90$ 
(6H, s*, 7-( $\left.\mathrm{CH}_{3}\right)_{2}$-sp.), $1.03\left(3 \mathrm{H}, \mathrm{d}, J=6.4 \mathrm{~Hz}, 9-\mathrm{CH}_{3}\right.$-sp.), 1.24-1.42 (2H, m, CH/CH ${ }_{2}$-sp.), $1.38(3 \mathrm{H}, \mathrm{d}, J=6.8 \mathrm{~Hz}$, 2- $\mathrm{CH}_{3}$-sp.), 1.62-1.80 (4H, m, CH/CH $\mathrm{CH}_{2}$-sp.), 3.87 (1H, s*, S-CH-sp.), 7.29, 7.30 (1H, 2dd, $J=8.8,2.0 \mathrm{~Hz}$, H6-ind.), $7.38\left(1 \mathrm{H}, \mathrm{t}^{*}, J=7.3 \mathrm{~Hz}, 3-\mathrm{C}_{6} \mathrm{H}_{5}(\mathrm{H} 4)\right.$-ind.), $7.45-7.48$ (3H, m, H4, 3- $\mathrm{C}_{6} \mathrm{H}_{5}(\mathrm{H} 3, \mathrm{H} 5)$-ind.), 7.50-7.54 (3H, m, H7, 3- $\mathrm{C}_{6} \mathrm{H}_{5}(\mathrm{H} 2, \mathrm{H} 6)$-ind.), 10.02 (1H, s, CONH), 12.13, 12.17 $(1 \mathrm{H}, 2 \mathrm{~s}, \mathrm{NH}) \mathrm{ppm} ;{ }^{13} \mathrm{C}$ NMR (APT, DMSO- $d_{6}, 125 \mathrm{MHz}$ ): $\delta=20.05\left(2-\mathrm{CH}_{3}\right.$-sp.), 22.23, 25.68, 26.21, $27.69\left(7-\left(\mathrm{CH}_{3}\right)_{2}\right.$, 9- $\mathrm{CH}_{3}$-sp.), 32.27 ( $\mathrm{CH}_{2}$-sp.), 36.84, 36.96 (C9-sp.), 37.46, 37.57 (C2-sp.), 45.22, 46.80, 47.29 ( $\mathrm{CH}_{2}$-sp., C7-sp.), 70.59 (C5-sp.), 114.56, 114.63 (CH-ar.), 118.52 (C-ar.), $119.29,119.38$ (CH-ar.), 124.58, 124.67 (CH-ar.), 125.44 (C-ar.), 127.44 (CH-ar.), 127.98 (C-ar.), 128.18, 128.24 (C-ar.), 128.97 (CH-ar.), 130.54 (CH-ar.), 133.46, 133.48 (C-ar.), 134.61 (C-ar.), 162.44 (CO-NH), 170.24 (CO-sp.) ppm; MS (ESI +): $m / z(\%)=497.1\left([\mathrm{M}+\mathrm{H}]^{+}, 31.2\right), 426.3$ $\left([(\mathrm{M}+\mathrm{H})+2]^{+}, 10.7\right)$.

5-Chloro- $\mathrm{N}$-(2-methyl-8-phenyl-3-oxo-1-thia-4-azaspiro[4.5]decan-4-yl)-3-phenyl- $1 \mathrm{H}$-indole-2-carboxamide (5h, $\left.\mathrm{C}_{30} \mathrm{H}_{28} \mathrm{CIN}_{3} \mathrm{O}_{2} \mathrm{~S}\right)$ Beige powder; yield: 82.3\%; m.p.: 213$215{ }^{\circ} \mathrm{C} ; R_{f}=0.69\left(\mathrm{EtOAc} / \mathrm{CHCl}_{3} 1: 1\right)$; IR (KBr): $\bar{v}=3289$ $(\mathrm{N}-\mathrm{H}), 1705,1663(\mathrm{C}=\mathrm{O}) \mathrm{cm}^{-1} ;{ }^{1} \mathrm{H}$ NMR (DMSO- $d_{6}$, $500 \mathrm{MHz}): \delta=1.45$ (3H, d, $J=6.8 \mathrm{~Hz}, 2-\mathrm{CH}_{3}$-sp.), 1.58-2.00 (8H, m, $\mathrm{CH}_{2}$-sp.), 2.45-2.51 (2H, m, CH-sp., DMSO-d6), $3.92\left(1 \mathrm{H}, \mathrm{d}^{*}, J=5.6 \mathrm{~Hz}, \mathrm{~S}-\mathrm{CH}-\mathrm{sp}.\right), 7.20\left(1 \mathrm{H}, \mathrm{tt}^{*}, J=7.3\right.$, $1.5 \mathrm{~Hz}, 8-\mathrm{C}_{6} \mathrm{H}_{5}(\mathrm{H} 4)$-sp.), $7.25\left(2 \mathrm{H}, \mathrm{d}^{*}, J=6.8 \mathrm{~Hz}, 8-\mathrm{C}_{6} \mathrm{H}_{5^{-}}\right.$ sp.), 7.30-7.34 (3H, m, H6-ind., 8- $\mathrm{C}_{6} \mathrm{H}_{5}$-sp.), $7.42\left(1 \mathrm{H}, \mathrm{tt}^{*}\right.$, $J=7.3,1.5 \mathrm{~Hz}, 3-\mathrm{C}_{6} \mathrm{H}_{5}(\mathrm{H} 4)$-ind.), 7.51-7.61 (6H, m, H4, H7, $3-\mathrm{C}_{6} \mathrm{H}_{5}(\mathrm{H} 2, \mathrm{H} 3, \mathrm{H} 5, \mathrm{H} 6)$-ind.), $10.21(1 \mathrm{H}, \mathrm{s}, \mathrm{CONH}), 12.18$ $(1 \mathrm{H}, \mathrm{s}, \mathrm{NH}) \mathrm{ppm} ;{ }^{13} \mathrm{C}$ NMR (APT, DMSO- $d_{6}, 125 \mathrm{MHz}$ ): $\delta=20.03\left(2-\mathrm{CH}_{3}\right.$-sp.), 30.76, $31.24\left(\mathrm{CH}_{2}\right.$-sp.), $37.38(\mathrm{C} 2-$ sp.), 38.10 ( $\mathrm{CH}_{2}$-sp.), 41.95 (C8-sp.), 70.78 (C5-sp.), 114.57, 114.63 (CH-ar.), 118.61 (C-ar.), 119.34, 119.43 (CH-ar.), 124.59, 124.69 (CH-ar.), 125.49 (C-ar.), 126.97 (8-ar.(CH)-sp.), 127.08 (CH-ar.), 127.43 (8-ar.(CH)-sp.), 127.91 (C-ar.), 128.27 (C-ar.), 128.95 (CH-ar.), 130.63 (CH-ar.), 133.45 (C-ar.), 134.63 (C-ar.), 146.28 (8-ar.(C)sp.), 162.14 (CO-NH), 170.47 (CO-sp.) ppm; MS (ESI-): $m / z(\%)=528.8\left([\mathrm{M}-\mathrm{H}]^{-}, 100\right), 530.8\left([(\mathrm{M}-\mathrm{H})+2]^{-}, 33.8\right)$.

\section{Antitubercular activity assays}

The microdilution method was performed according to a standard protocol from the Clinical and Laboratory Standard Institute (CLSI) [27, 28]. The resazurin microtitre assay (REMA) has been developed as a colorimetric and standard method for drug susceptibility testing. The minimum inhibitory concentrations (MICs) were determined according to color changes at the end of incubation [29-31]. The strain used, i.e., Mycobacterium tuberculosis H37Rv ATCC 27294 is susceptible to all common antimycobacterial drugs.
Middlebrook 7H9 broth medium (Becton and Dickinson, USA) was used and the medium was adjusted to $\mathrm{pH} 7.0$ at $25^{\circ} \mathrm{C}$. Each bottle was controlled for sterility before it was used. Resazurin purchased from Sigma-Aldrich (St Louis, USA) was dissolved in sterile distilled water to a final concentration of $0.02 \%$ and sterilized by filtration, then stored at $4{ }^{\circ} \mathrm{C}$ until use. Rifampicin purchased from Becton-Dickinson (BD, USA) was dissolved in sterile distilled water to a final concentration of $1 \mu \mathrm{g} / \mathrm{cm}^{3}$ (critical concentration). The synthesized compounds were dissolved in $100 \%$ dimethyl sulfoxide according to CLSI methods [27, 28]. Stock solutions were obtained by 40 -fold dilution in DMSO followed by sterile filtration. From here, working stocks at $4000 \mu \mathrm{M}$ were obtained by diluting 1/10 in MB7H9 medium. The final concentrations were $1000 \mu \mathrm{M}$ to $0.49 \mu \mathrm{M}$ for the synthesized compounds. For rifampicin, the critical concentration $(1 \mu \mathrm{g} /$ $\mathrm{cm}^{3}$ ) was used [27, 28].

Inoculum suspensions of mycobacteria were prepared according to the CLSI guidelines as described previously. The isolates were subcultured on Löwenstein Jensen medium and incubated at $37{ }^{\circ} \mathrm{C}$ for $20-25$ days. A few colonies from freshly grown $M$. tuberculosis were suspended in Middlebrook $7 \mathrm{H} 9$ broth medium to obtain $1.0 \mathrm{McFarland}$ turbidity, then diluted ten times with the same medium.

The broth microdilution test was performed in sterile 96-well U-shaped microdilution plates (LP Italiano SPA, Milano, Italy). Rows A-F contained $100 \mathrm{~mm}^{3}$ of the compound dilutions, whereas rows $\mathrm{G}$ (positive control) and $\mathrm{H}$ (negative control) contained $100 \mathrm{~mm}^{3}$ medium. One hundred $\mathrm{mm}^{3}$ of the corresponding inoculum was added to all wells except for row $\mathrm{H}$. The microplates were incubated at $35^{\circ} \mathrm{C}$ for about 7-10 days, when mycobacterial growth was clearly visible as a white sediment in the positive control. Microbial growth was confirmed by Ehrlich-Ziehl-Neelsen acid-fast stain. Resazurin solution $\left(30 \mathrm{~mm}^{3}\right)$ was added to each well and the plates were incubated for one additional day. At that time, the first purple colored well in which no growth was visible, was defined as the compounds' MIC value (Table 1).

\section{Antiviral activity assays}

Stock solutions of the test and reference compounds were prepared in $100 \%$ DMSO at 5-25 mM. During incubation with the cells, the highest test concentration was $100 \mu \mathrm{M}$ (or $250 \mu \mathrm{M}$ for ribavirin). The antiviral reference compounds were: ganciclovir, brivudin, zanamivir, amantadine, ribavirin, dextran sulfate-10,000, and mycophenolic acid. Antiviral evaluation was carried out with a broad panel of viruses using cytopathic effect (CPE) reduction assays. Human influenza A (H1N1 and H3N2) and B viruses were examined on Madin-Darby canine kidney (MDCK) cells. Respiratory syncytial virus, vesicular stomatitis virus and Coxsackie B4 virus were evaluated on human cervix carcinoma 
HeLa cells. African Green Monkey Vero cells were used for parainfluenza-3 virus, reovirus-1, Sindbis virus, Coxsackie B4 virus, Punta Toro virus and yellow fever virus. Human embryonic lung (HEL) fibroblast cells were infected with herpes simplex virus types 1 and 2, vaccinia virus, human adenovirus-2, and human coronavirus 229E.

Semiconfluent cell cultures in 96-well plates were infected with virus at a multiplicity of infection of 100 $\mathrm{CCID}_{50}(50 \%$ cell culture infective dose) per well. Together with the virus, fourfold dilutions of the test or reference compounds were added. The plates were incubated at $37^{\circ} \mathrm{C}$ (or $35^{\circ} \mathrm{C}$ for influenza- and coronavirus) until far advanced CPE was visible, i.e., during 3-6 days or 10 days in the case of adenovirus-2. Then, microscopy was performed to score the CPE and calculate the 50\% antivirally effective concentration $\left(\mathrm{EC}_{50}\right)$. Microscopy was also done to assess cytotoxicity, expressed as the compound concentration causing minimal changes in cell morphology (minimal cytotoxic concentration; MCC).

\section{Antibacterial and antifungal activity assays}

Antimicrobial activity against Pseudomonas aeruginosa ATCC 27853, Escherichia coli ATCC 25922, Klebsiella pneumoniae ATCC 4352, Proteus mirabilis ATCC 14153, Staphylococcus aureus ATCC 29213, Staphylococcus epidermidis ATCC 12228, Enterococcus faecalis ATCC 29212, Candida albicans ATCC 10231, Candida parapsilosis ATCC 22019, and Candida tropicalis ATCC 750 was determined by the microbroth dilutions technique using CLSI recommendations [32,33]. Serial twofold dilutions ranging from $2500 \mu \mathrm{M}$ to $1.22 \mu \mathrm{M}$ were prepared in the test medium, i.e., Mueller-Hinton broth for bacteria and RPMI-1640 medium for yeast strains. The inoculum was prepared using a 4-6 h broth culture of each bacteria type and $24 \mathrm{~h}$ culture of yeast strains adjusted to a turbidity equivalent to 0.5 McFarland standard, diluted in broth media to give a final concentration in the test tray of $5 \times 10^{5} \mathrm{cfu} / \mathrm{cm}^{3}$ for bacteria and $5 \times 10^{3} \mathrm{cfu} / \mathrm{cm}^{3}$ for yeast. The trays were covered and placed into plastic bags to prevent evaporation. The bacteria trays were incubated at $35^{\circ} \mathrm{C}$ for $18-20 \mathrm{~h}$ while the yeastcontaining trays were incubated at $35^{\circ} \mathrm{C}$ for $46-50 \mathrm{~h}$. The MIC was defined as the lowest concentration of compound giving complete inhibition of visible growth. Amikacin and fluconazole were used as reference antibiotics for bacteria and yeast, respectively; their MIC values were within the accuracy range of the CLSI guidelines [34].

Acknowledgements This work was supported in part by the Research Fund of Istanbul University (Project Number BYP-57695). LN acknowledges dedicated assistance from Leentje Persoons and her team members.

\section{References}

1. World Health Organization (WHO) (2017) Global Tuberculosis Report. http://www.who.int/tb/publications/global_report/en/. Accessed 10 June 2018

2. Streicher EM, Müller B, Chihota V, Mlambo C, Tait M, Pillay M, Trollip A, Hoek KGP, Sirgel FA, Pittius NCG, Helden PD, Victor TC, Warren RM (2012) Infect Gene Evol 12:686

3. Zumla AI, Gillespie SH, Hoelscher M, Philips PP, Cole ST, Abubakar I, McHugh TD, Schito M, Maeurer M, Nunn AJ (2014) Lancet Infect Dis 14:327

4. Zignol M, Dean AS, Falzon D, Gemert W, Wright A, Deun A, Portaels F, Laszlo A, Espinal MA, Pablos-Méndez A, Bloom A, Aziz MA, Weyer K, Jaramillo E, Nunn P, Floyd K, Raviglione MC (2016) N Engl J Med 375:1081

5. Ankale P, Nair G, Uppe A, Mathew A, Shah R (2017) Eur Respir J 50:PA2727

6. Wallis RS, Maeurer M, Mwaba P, Chakaya J, Rustomjee R, Migliori GB, Marais B, Schito M, Churchyard G, Swaminathan S, Hoelscher M, Zumla A (2016) Lancet Infect Dis 16:e34

7. Poce G, Cocozza M, Consalvi S, Biava M (2014) Eur J Med Chem $86: 335$

8. Chetty S, Ramesh M, Singh-Pillay A, Soliman MES (2017) Bioorg Med Chem Lett 27:370

9. Onajole OK, Pieroni M, Tipparaju SK, Lun S, Stec J, Chen G, Gunosewoyo H, Guo H, Ammerman NC, Bishai WR, Kozikowski AP (2013) J Med Chem 56:4093

10. Lun S, Guo H, Onajole OK, Pieroni M, Gunosewoyo H, Chen G, Tipparaju SK, Ammerman NC, Kozikowski AP, Bishai WR (2013) Nat Commun 4:2907

11. Kondreddi RR, Jiricek J, Rao SP, Lakshminarayana SB, Camacho LR, Rao R, Herve M, Bifani P, Ma NL, Kuhen K, Goh A, Chatterjee AK, Dick T, Diagana TT, Manjunatha UH, Smith PW (2013) J Med Chem 56:8849

12. Rao SP, Lakshminarayana SB, Kondreddi RR, Herve M, Camacho LR, Bifani P, Kalapala SK, Jiricek J, Ma NL, Tan BH, Ng SH, Nanjundappa M, Ravindran S, Seah PG, Thayalan P, Lim SH, Lee BH, Goh A, Barnes WS, Chen Z, Gagaring K, Chatterjee AK, Pethe K, Kuhen K, Walker J, Feng G, Babu S, Zhang L, Blasco F, Beer D, Weaver M, Dartois V, Glynne R, Dick T, Smith PW, Diagana TT, Manjunatha UH (2013) Sci Transl Med 5:214ra168

13. Stec J, Onajole OK, Lun S, Guo H, Merenbloom B, Vistoli G, Bishai WR, Kozikowski AP (2016) J Med Chem 59:6232

14. Franz ND, Belardinelli JM, Kaminski MA, Dunn LC, Moura VCN, Blaha MA, Truong DD, Li W, Jackson M (2017) Bioorg Med Chem 25:3746

15. Cihan-Üstündă̆ G, Çapan G (2012) Mol Divers 16:525

16. Güzel Ö, Terzioğlu N, Çapan G, Salman A (2006) Arkivoc xii:98

17. Cihan-Üstündağ G, Şatana D, Özhan G, Çapan G (2015) J Enzyme Inhib Med Chem 31:369

18. Vanderlinden E, Göktaş F, Cesur Z, Froeyen M, Reed ML, Russell CJ, Cesur N, Naesens L (2010) J Virol 84:4277

19. Göktaş F, Vanderlinden E, Naesens L, Cesur N, Cesur Z (2012) Bioorg Med Chem 20:7155

20. Göktaş F, Vanderlinden E, Naesens L, Cesur Z, Cesur N, Taş P (2015) Phosphorus. Sulfur Silicon Relat Elem 190:1075

21. Yamamoto H, Inaba S, Okamoto T, Hirohashi T, Ishizumi K, Yamamoto M, Maruyama I, Mori K, Kobayashi T (1969) Tranquilizer benzodiazepine derivatives. South African Patent ZA 6803041 A, Jan 18, 1969; (1969) Chem Abstr 71:124519

22. Abdelrahman MH, Aboraia AS, Youssif BGM, Elsadek BEM (2017) Chem Biol Drug Des 90:64

23. Hiremath SP, Mruthyunjayaswamy BHM, Purohit MG (1978) Indian J Chem 16B:789 
24. Ergenç N, Günay NS, Demirdamar R (1998) Eur J Med Chem 33:143

25. Kline T, Andersen NH, Harwood EA, Bowman J, Malanda A, Endsley S, Erwin AL, Doyle M, Fong S, Harris AL, Mendelsohn B, Mdluli K, Raetz CRH, Stover CK, Witte PR, Yabannavar A, Zhu S (2002) J Med Chem 45:3112

26. Kocabalkanlı A, Cihan-Üstündağ G, Naesens L, Mataracı-Kara E, Nassozi M, Çapan G (2017) Arch Pharm Chem Life Sci 350:1

27. Amsterdam D (2005) In: Lorian V (ed) Antibiotics in laboratory medicine, 5th edn. Williams and Wilkins, Philadelphia

28. Clinical and Laboratory Standards Institute (2011) Susceptibility testing of mycobacteria, nocardiae and other aerobic actinomycetes. Approved Standard Second Edition M24-A2 Clinical and Laboratory Standards Institute, Wayne

29. Martin A, Portaels F, Palomino JC (2007) J Antimicrob Chemother 59:175
30. Martin A, Panaiotov S, Portaels F, Hoffner S, Palomino JC, Angeby K (2008) J Antimicrob Chemother 62:56

31. Coban AY, Akbal AU, Uzun M, Durupinar B (2015) Mem Inst Oswaldo Cruz 110:649

32. CLSI document (1997) Approved standard-2nd edition M27-A2. Clinical and Laboratory Standards Institute, Wayne

33. CLSI document (2006) Approved standard-7th edition M7-A7. Clinical and Laboratory Standards Institute, Wayne

34. CLSI document (2010) 7th informational supplement M100-S20. Clinical and Laboratory Standards Institute, Wayne

Publisher's Note Springer Nature remains neutral with regard to jurisdictional claims in published maps and institutional affiliations. 